\section{LAUR. $96-4639$ APRnoved for public release;}

The Effects of Pseudo Magnetic Fields in Molecular Spectra and Scattering

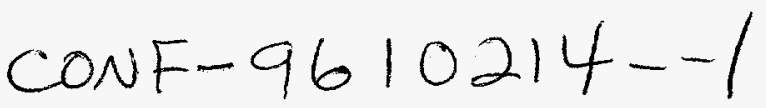

Author(s):

Submitted to:

Brian K. Kendrick

bitas

$13 \sqrt{3}$

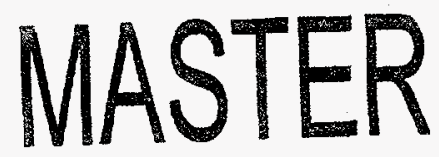

The International Journal of Quantum Chemistry: Proceedings of "Properties of Molecules in strong Magnetic Fields Workshop", Oct. 24-25, 1996, The University of Florida, FL.

Los Alamos National Laboratory, an affirmative action/equal opportunity employer, is operated by the University of California for the U.S. Department of Energy under contract W-7405-ENG-36. By acceptance of this article, the publisher recognizes that the U.S. Government retains a nonexclusive, royalty-free license to publish or reproduce the published form of this contribution, or to allow others to do so, for U.S. Government purposes. Los Alamos National Laboratory requests that the publisher identify this article as work performed under the auspices of the U.S. Department of Energy. The Los Alamos National Laboratory strongly supports academic freedom and a researcher's right to publish; as an institution, however, the Laboratory does not endorse the viewpoint of a publication or guarantee its fechnical correctness. 


\section{DISCLAIMER}

This report was prepared as an account of work sponsored by an agency of the United States Government. Neither the United States Government nor any agency thereof, nor any of their employees, makes any warranty, express or implied, or assumes any legal liability or responsibility for the accuracy, completeness, or usefulness of any information, apparatus, product, or process disclosed, or represents that its use would not infringe privately owned rights. Reference herein to any specific commercial product, process, or service by trade name, trademark, manufacturer, or otherwise does not necessarily constitute or imply its endorsement, recommendation, or favoring by the United States Government or any agency thereof. The views and opinions of authors expressed herein do not necessarily state or reflect those of the United States Government or any agency thereof. 


\section{DISCLAMMER}

Portions of this document may be illegible in electronic image products. Images are produced from the best available original document. 


\title{
The Effects of Pseudo Magnetic Fields in Molecular Spectra and Scattering
}

\author{
Brian Kendrick \\ Theoretical Division (T-12, MS-B268) \\ Los Alamos National Laboratory \\ Los Alamos, New Mexico 87545
}

(December 2, 1996)

\begin{abstract}
Pseudo magnetic fields appear in the Born-Oppenheimer method for molecules when conical intersections or electronic angular momenta are taken into account. These fields are not real magnetic fields but they have the same mathematical properties and can lead to real observable effects in the dynamics of molecules. A general vector potential (gauge theory) approach for including these field effects in the Born-Oppenheimer method is introduced and applied to $\mathrm{H}+\mathrm{O}_{2}$ scattering and the vibrational spectrum of $\mathrm{Na}_{3}(\mathrm{X})$ for zero total angular momentum $(J=0)$. The scattering results for $\mathrm{HO}_{2}$ show significant shifts in the resonance energies and lifetimes due to a "magnetic solenoid" type field originating from the $C_{2 v}$ conical intersection in $\mathrm{HO}_{2}$. Significant changes in the state-to-state transition probabilities are also observed. The non-degenerate $A_{1}$ and $A_{2}$ vibrational spectra of $\mathrm{Na}_{3}(\mathrm{X})$ show significant shifts in the energy levels due to a "magnetic solenoid" type field originating from the $D_{3 h}$ conical intersection in $\mathrm{Na}_{3}$. These two examples show that the effects of pseudo magnetic fields can be significant and
\end{abstract}


in many cases they must be included in order to obtain agreement between theory and experiment. The newly developed gauge theory techniques for treating pseudo magnetic fields are also relevant for including the effects of real magnetic fields.

\section{INTRODUCTION}

In the Born-Oppenheimer treatment of molecules the adiabatic electronic wavefunction depends parametrically on the nuclear coordinates. In the standard application of this approach, the nuclear dependence of the electronic wavefunction is initially ignored, and it is assumed that the effects due to the nuclear motion can be added in a perturbative fashion. ${ }^{1}$ However, in the presence of a conical intersection this is not at all the case. In 1963 Herzberg and Longuet-Higgins ${ }^{2}$ showed that a real electronic wavefunction is doublevalued (i.e. changes sign) when the nuclear coordinates traverse a closed path encircling a conical intersection. This sign change is certainly not a small effect and signifies that the standard Born-Oppenheimer (BO) treatment needs to be generalized. In 1979 Mead and Truhlar ${ }^{3}$ generalized the BO method by showing that the sign change can be accounted for by introducing a vector potential (or gauge potential) into the nuclear Schrödinger equation. In their approach the real double-valued electronic wavefunction is multiplied by a complex phase factor which changes sign on encircling the conical intersection so that the resulting complex electronic wavefunction is single-valued. However, in going to a complex singlevalued electronic wavefunction, the nuclear Schrödinger equation acquires a vector potential. The resulting differential equations are identical to those of a charged particle moving in the presence of a magnetic solenoid, and Mead later called this effect the "molecular AharonovBohm effect". "In 1984 , Berry ${ }^{5}$ considered a general quantum system with parametric time dependence undergoing adiabatic time evolution. He showed that the "molecular AharonovBohm effect" was a special case of the more general geometric phase often referred to as "Berry's Phase". Berry's influential paper generated much theoretical interest in this effect. 
However, even though the geometric phase was first discovered in molecules many years ago, it was not included in molecular scattering and bound state calculations until recently. ${ }^{6-11}$ Part of this delay was due to the fact that only in the past five to ten years have the necessary numerical techniques and computer resources become available.

It is important to realize that the magnetic fields which appear in the generalized BornOppenheimer method and give rise to the geometric phase are not real magnetic fields. They are pseudo magnetic fields which represent internal coupling between the electronic and nuclear degrees of freedom. However, since the resulting equations are formally equivalent to those of electromagnetic theory, the theoretical and computational techniques that are used to treat pseudo magnetic fields can be used to treat molecules in real magnetic fields.

The standard Born-Oppenheimer method which neglects the coupling between the electronic and nuclear motion is briefly reviewed in Section II. Conical intersections and electronic angular momentum are introduced in Section III and their treatment using a generalized Born-Oppenheimer method discussed. Some results of recent $\mathrm{H}+\mathrm{O}_{2}$ scattering calculations for zero total angular momentum $(J=0)$ which include the effects of the geometric phase are presented in Section IV. The first calculations to include the effects of the geometric phase in the vibrational spectrum of $\mathrm{Na}_{3}(\mathrm{X})$ are presented in Section $\mathrm{V}$. These results clearly show that pseudo magnetic fields give rise to significant dynamical effects (i.e. the geometric phase) and must be included in order to obtain the correct results and agreement with experiment. The application of these techniques to real magnetic fields and some conclusions are discussed in Section VI.

\section{THE STANDARD BORN-OPPENHEIMER METHOD}

The molecular Schrödinger equation is given by

$$
H \Psi_{\mathrm{tot}}=E \Psi_{\mathrm{tot}},
$$

where $\Psi_{\text {tot }}$ is the total molecular wavefunction, $H$ the total molecular Hamiltonian and $E$ the total energy. We restrict our present treatment to triatomic molecules (i.e. $\mathrm{HO}_{2}, \mathrm{Na}_{3}$, 
etc.). The six nuclear coordinates relative to the center of mass consist of three internal and three orientational coordinates. The three internal coordinates are taken to be the hyperspherical coordinates $(\rho, \theta, \phi)$ of Smith-Johnson ${ }^{12,13}$ except that the angle $\phi$ is related to the angle $\chi$ of Pack-Parker ${ }^{14}$ by $\phi=2 \chi$. The $(\rho, \theta, \phi)$ are functions of the three internuclear distances. The three orientational coordinates are taken to be the Euler angles which give the orientation of the body-frame (BF) relative to the space-frame (SF). We use APH BF axes fixed on the instantaneous principal axes of inertia. ${ }^{14}$ We differentiate between the two types of coordinates by expressing $\mathrm{x}=(\mathrm{x}, \hat{x})$ with $\mathrm{x}=(\rho, \theta, \phi)$ denoting the three internal coordinates and $\hat{x}=(\alpha, \beta, \gamma)$ the three Euler angles.

After separating the center of mass motion, we can express $H$ in SF coordinates as

$$
H \equiv-\frac{\hbar^{2}}{2 \mu} \nabla^{2}+h(\mathrm{x}),
$$

where $\nabla^{2}$ is the 6 -dimensional Laplacian with respect to the six nuclear coordinates $\mathbf{x}, \mu$ is the three body reduced mass $\mu \equiv\left(m_{\mathrm{A}} m_{\mathrm{B}} m_{\mathrm{C}} /\left(m_{\mathrm{A}}+m_{\mathrm{B}}+m_{\mathrm{C}}\right)\right)^{\frac{1}{2}}$, and $h(\mathrm{x})$ is the electronic Hamiltonian which depends parametrically on the three internal nuclear coordinates. We note that in the presence of real magnetic fields, the separation of the center of mass motion is non-trivial and a more general treatment is necessary. ${ }^{15,16}$

As usual, we assume that the electronic part of the problem has been solved for the electronic energy eigenvalues $V_{n}$ and eigenfunctions $\varphi_{n}$ in BF coordinates

$$
h(\mathrm{x}) \varphi_{n}(\mathrm{r} ; \mathrm{x})=V_{n}(\mathrm{x}) \varphi_{n}(\mathrm{r} ; \mathrm{x})
$$

where $\mathbf{r}$ denotes all of the electronic coordinates relative to the BF. The eigenvalues and eigenfunctions both depend parametrically on the three internal nuclear coordinates $\mathrm{x}$. We neglect all internal magnetic interactions (i.e. spin-orbit) and consider only coulomb interactions between the various constituents. Thus, the electronic Hamiltonian is real with real eigenvalues and electronic eigenfunctions. In the presence of real external magnetic fields, the electronic structure of a molecule is significantly altered and new ab initio techniques must be used. ${ }^{17}$ Only simple diatomic molecules with hydrogen atoms have been treated in detail. ${ }^{17-20}$ 
The total molecular wavefunction can be expanded in terms of a complete set (or almost complete set) of electronic eigenfunctions

$$
\Psi_{\mathrm{tot}}=\sum_{n=0}^{N} \Psi_{n}(\mathrm{x}) \varphi_{n}(\mathrm{r} ; \mathrm{x}) \psi^{\mathcal{N}},
$$

where $N$ is in general infinite, the expansion coefficients $\Psi_{n}(\mathbf{x})$ are the "nuclear" wavefunctions and $\psi^{\mathcal{N}}$ is the nuclear spin wavefunction. For low energies and a non-degenerate ground electronic state, we can neglect the coupling to excited electronic states and truncate the sum in Eq. 4 to only one term

$$
\Psi_{\text {tot }} \approx \Psi_{0}(\mathbf{x}) \varphi_{0}(\mathbf{r} ; \mathbf{x}) \psi^{\mathcal{N}}
$$

where $n=0$ denotes the ground electronic state. For degenerate states and higher energies, more terms in the sum over $n$ must be included. In the present work Eq. 5 is valid, and we drop the subscript on the nuclear and electronic wavefunctions with the understanding that they denote the ground electronic state.

If one substitutes Eq. 5 into Eq. 1 , multiplies on the left by $\varphi(r ; x)$, integrates over $\mathbf{r}$ and uses Eq. 3, one obtains the standard Born-Oppenheimer (BO) equation for the nuclear motion in the SF

$$
\left[-\frac{\hbar^{2}}{2 \mu} \nabla^{2}+V(\mathrm{x})\right] \Psi(\mathrm{x})=E \Psi(\mathrm{x}) .
$$

This usual BO result neglects the electronic angular momentum and ignores geometric phase

effects. We note that in the derivation of Eq. 6 we used the fact that $\langle\varphi(\mathrm{x})|\nabla| \varphi(\mathrm{x})\rangle=0$ which is straightforward to prove by differentiating the normalization equation $\langle\varphi(\mathrm{x}) \mid \varphi(\mathrm{x})\rangle=1$ and using the fact that $|\varphi(\mathrm{x})\rangle$ is real.

\section{THE GENERALIZED BORN-OPPENHEIMER METHOD}

We now generalize Eq. 6 to include geometric phase effects. The real ground state electronic eigenfunction $\varphi(\mathbf{r} ; \mathrm{x})$ changes sign whenever the nuclear motion encircles a conical 
intersection between the ground and first excited electronic states. ${ }^{2}$ Even if the actual intersection lies very high in energy, it is important to realize that the sign change (geometric phase) occurs for much lower energies. The scattering or bound state energy only needs to be high enough so that the nuclear motion can encircle the intersection. For systems with identical nuclei such as $\mathrm{HO}_{2}$ and $\mathrm{Na}_{3}$, the sign change of the electronic wavefunction corresponds to a change in permutation symmetry. In order for the total wavefunction $\Psi_{\text {tot }}$ of Eq. 5 to remain single-valued and maintain the correct permutation symmetry for all nuclear configurations, the nuclear wavefunction $\Psi(\mathrm{x})$ must also change sign. There is no built-in mechanism to ensure that the nuclear wavefuntion changes sign in the conventional BO approach of Eq. 6 .

We denote the $d$ dimensional internal nuclear parameter space by $\mathcal{M}(d=3$ for triatomics). The subspace of $\mathcal{M}$ for which the ground and first excited state electronic eigenvalues become degenerate is denoted by $\mathcal{D} \subset \mathcal{M}$ which is of dimension $d-2 . .^{21,22}$ Thus, for triatomic molecules the degeneracy manifold $\mathcal{D}$ is a $1 \mathrm{D}$ curve in the $3 \mathrm{D}$ nuclear parameter space $\mathcal{M}$. The two conditions $u(\mathrm{x})=0$ and $v(\mathrm{x})=0$ which define $\mathcal{D}$ can be expressed in terms of any two diagonal cofactors of the matrix $\mathbf{w}(\mathrm{x}) \equiv \mathrm{h}(\mathrm{x})-\mathbf{I} V_{0}(\mathrm{x})$ where $\mathrm{h}$ is the electronic Hamiltonian and $V_{0}$ is the ground state eigenvalue. ${ }^{22}$ In an infinitesimal region near $\mathcal{D}$, the relevant part of the electronic Hamiltonian is a $2 \times 2$ matrix of the form ${ }^{3,21}$

$$
h(\mathrm{x})=-\left(\begin{array}{cc}
\delta u & \delta v \\
\delta v & -\delta u
\end{array}\right)=-r\left(\begin{array}{cc}
\cos \eta & \sin \eta \\
\sin \eta & -\cos \eta
\end{array}\right),
$$

where $\delta u(\mathrm{x})$ and $\delta v(\mathrm{x})$ are infinitesimal displacements from the origin in the 2D Cartesian space spanned by $u \mathbf{e}_{u}$ and $v \mathbf{e}_{v}$ (see Fig. 1). The polar coordinates are denoted by $(r, \eta)$ where $r$ is the radial distance from the origin in $u v$ space and $\eta$ is the azimuthal angle around the origin.

Almost by inspection we can write down the eigenvalues and eigenvectors of the electronic Hamiltonian matrix of Eq. 7. The two eigenvalues are $\pm r$ which correspond to the upper and lower cones in Fig. 1. The two corresponding eigenvectors are given by 


$$
\varphi^{+}=\left(\begin{array}{c}
-\sin \frac{\eta}{2} \\
\cos \frac{\eta}{2}
\end{array}\right) \quad, \quad \varphi^{-}=\left(\begin{array}{c}
\cos \frac{\eta}{2} \\
\sin \frac{\eta}{2}
\end{array}\right) .
$$

It is clear that, due to the half-angle functional dependence upon the azimuthal angle $\eta$, these eigenvectors change sign (i.e. are double-valued) for any closed path in the nuclear parameter space for which $\eta$ changes by $2 \pi$. Equation 8 is valid only in the infinitesimal region near the conical intersection. However, the sign change (double-valuedness) exists globally. That is, any closed path in $\mathcal{M}$ which encircles $\mathcal{D}$ (no matter how far away from $\mathcal{D}$ ) results in a sign change in the adiabatic ground state electronic eigenvector. ${ }^{23}$ The global expression for the angle $\eta$ is given by $\eta(\mathrm{x}) \equiv \tan ^{-1}(v(\mathrm{x}) / u(\mathrm{x}))$ which is in general a complicated function of the three internal nuclear degrees of freedom. ${ }^{24}$ Recently, techniques have been developed to explicitly compute $\eta(\mathrm{x})$ for a general polyatomic molecule. ${ }^{22}$ However, for treating single conical intersections in triatomic molecules (such as in $\mathrm{HO}_{2}$ and $\mathrm{Na}_{3}$ ) an explicit functional form for $\eta(\mathrm{x})$ can often be derived analytically. ${ }^{8}$ It is important to realize that the functional forms of $u(\mathrm{x})$ and $v(\mathrm{x})$ are not unique. Hence, $\eta(\mathrm{x})$ is not unique. The only requirement is that $\eta(\mathrm{x})$ change by $2 \pi$ for any closed path in $\mathcal{M}$ which encircles $\mathcal{D}$. This freedom in choosing $\eta$ is called "gauge freedom" and different choices for $\eta$ are related by $U(1)$ gauge transformations. ${ }^{8,22}$

Following Mead and Truhlar ${ }^{3}$, we multiply the real adiabatic (ground state) BF electronic wavefunction by a complex phase

$$
\varphi^{\mathcal{C}}(\mathbf{r} ; \mathrm{x}) \equiv \exp \left(i \frac{l}{2} \eta(\mathrm{x})\right) \varphi(\mathbf{r} ; \mathrm{x}),
$$

where $l$ is an odd integer and $\eta(\mathrm{x})$ is the azimuthal angle discussed above which changes by $2 \pi$ for any nuclear motion which encircles a conical intersection. The complex phase factor cancels the sign change arising from the real electronic wavefunction $\varphi(r ; x)$ giving rise to a complex continuous single-valued electronic wavefunction $\varphi^{\mathcal{C}}(\mathrm{r} ; \mathrm{x})$. It is straightforward to show that the different choices for $l$ are related by gauge transformations. ${ }^{3}$ Hence, the choice of $l$ is matter of convenience although larger values of $l$ make convergence more difficult.

If we do not neglect the electronic angular momentum, then the space-frame (SF) elec- 
tronic wavefunction in not equal to the body-frame (BF) electronic wavefunction. The complex SF electronic wavefunction, $\varphi^{\mathcal{C}}(\mathbf{r} ; \mathbf{x})$, is related to the complex BF electronic wavefunction of Eq. 9 via

$$
\varphi^{\mathcal{c}}(\mathbf{r} ; \mathbf{x}) \equiv U(\alpha, \beta, \gamma) \varphi^{\mathcal{c}}(\mathbf{r} ; \mathbf{x})
$$

where $U$ is the rotation operator which transforms the electronic wavefunctions in the $\mathrm{BF}$ to the SF. It is explicitly defined as ${ }^{25,26}$

$$
U(\alpha, \beta, \gamma) \equiv e^{-i \mathcal{J}_{z} \alpha} e^{-i \mathcal{J}_{y} \beta} e^{-i \mathcal{J}_{z} \gamma}
$$

where the $\mathcal{J}_{i}$ are the components of the total electronic angular momentum in the BF.

Using Eqs. 10 and 9, we repeat the same steps which lead to Eq. 6 to obtain the generalized $\mathrm{BO}$ equation for the nuclear motion m $^{3,8,21,27}$

$$
\left[\frac{\hbar^{2}}{2 \mu}(-i \nabla-\mathbf{A}(\mathbf{x}))^{2}+\varepsilon(\mathbf{x})\right] \Psi^{\mathcal{c}}(\mathbf{x})=E \Psi^{\mathcal{c}}(\mathbf{x})
$$

where the complex nuclear wavefunctions $\Psi^{\mathcal{C}}(\mathbf{x})$ are single-valued and $\mathbf{A}(\mathbf{x})$ is the vector potential (or $U(1)$ gauge potential) defined $a s^{3,8,21,27}$

$$
\mathbf{A}(\mathbf{x}) \equiv i\left\langle\varphi^{\mathcal{C}}(\mathbf{x})\right|\left(\nabla\left|\varphi^{\mathcal{C}}(\mathbf{x})\right\rangle\right)
$$

and $\varepsilon(\mathbf{x})$ is the effective scalar potential defined $a s^{8,27}$

$$
\varepsilon(\mathbf{x}) \equiv V(\mathrm{x})-\frac{\hbar^{2}}{2 \mu} \sum_{m \neq 0}\left\langle\varphi_{0}^{\mathcal{C}}(\mathbf{x})\right|\left(\nabla\left|\varphi_{m}^{\mathcal{C}}(\mathbf{x})\right\rangle\right) \cdot\left\langle\varphi_{m}^{\mathcal{C}}(\mathbf{x})\right|\left(\nabla\left|\varphi_{0}^{\mathcal{C}}(\mathbf{x})\right\rangle\right)
$$

By factoring the gradient operator as $\nabla=\nabla_{\mathbf{x}}+\nabla_{\hat{x}}$, we can express $\mathbf{A}(\mathbf{x})$ as

$$
\mathbf{A}(\mathbf{x})=\mathbf{A}(\mathrm{x})+\mathbf{A}(\mathrm{x}, \hat{x})
$$

where

$$
\begin{aligned}
\mathbf{A}(\mathrm{x}) & \equiv-\frac{l}{2} \nabla_{\mathrm{x}} \eta(\mathrm{x}) \\
\mathbf{A}(\mathrm{x}, \hat{x}) & \equiv i\left\langle\varphi(\mathrm{x})\left|U^{-1}(\hat{x})\left(\nabla_{\hat{x}} U(\hat{x})\right)\right| \varphi(\mathrm{x})\right\rangle
\end{aligned}
$$


Equation 16 has the same mathematical form as the vector potential of a magnetic solenoid located at the conical intersection. ${ }^{3,4}$ By taking the curl of Eq. 16, we find that the resulting "magnetic". field is zero everywhere except at the conical intersection where it has a delta function singularity ${ }^{3}$

$$
\mathbf{B}(\mathrm{x}) \equiv \vec{\nabla}_{\mathrm{x}} \times \mathbf{A}(\mathrm{x})=-l \pi \delta(\mathrm{x}) \mathbf{e}_{z}
$$

where $\mathbf{e}_{z}$ points along the $z$ axis perpendicular to the $u v$-plane with the conical intersection located at the origin (see Fig. 1)..$^{3,21}$

The real nuclear wavefunctions $(\Psi)$ are constructed by applying the appropriate symmetry projection operators and phase factors to the complex $\Psi^{\mathcal{C}}$ (see Section IV). ${ }^{3,8}$ The change in "phase" of a real nuclear wavefunction for any closed path $C$ in the nuclear parameter space is given by

$$
\Psi_{f}=\exp \left[\oint_{C} \mathbf{A} \cdot \mathrm{dl}\right] \Psi_{i}
$$

where $\Psi_{i}$ and $\Psi_{f}$ denote the initial and final real nuclear wavefunctions, respectively. By using Stokes's theorem, we can express the line integral of $\mathbf{A}$ as a surface integral of $\mathbf{B}$ which shows that the geometric phase $\left(\beta_{g}\right)$ is equal to the "flux". of the pseudo magnetic field through the surface $S$ enclosed by $C$

$$
\beta_{g} \equiv \oint_{C} \mathbf{A} \cdot \mathrm{d} \mathbf{l}=\int_{S} \mathbf{B} \cdot \mathrm{ds}
$$

For any closed path $C$ which encircles the intersection, we find that $\beta_{g}=-l \pi$. That is, the real nuclear wavefunction changes sign (is double-valued) around the conical intersection. For any closed path $C$ which does not encircle the intersection, we find that $\beta_{g}=0$ and the real nuclear wavefunction is single-valued (as expected).

The angular part of the vector potential is given by Eq. 17 and involves matrix elements of the electronic angular momentum. Upon substituting the expression for $U$ given by Eq. 11 into Eq. 17 and using the commutation relations of the $\mathcal{J}_{i}$, an explicit expression for the $\mathbf{A}(\mathrm{x}, \hat{x})$ can be derived. ${ }^{8}$ The resulting vector potential is similar in form to that of a magnetic monopole. ${ }^{27-30}$ 
Using the expression for $\left|\varphi_{0}^{\mathcal{C}}(\mathbf{x})\right\rangle$ given in Eqs. 10 and 9, we can express the factors which appear in the effective potential $\varepsilon(\mathbf{x})$ as (for $m \neq 0$ )

$$
\left\langle\varphi_{0}^{\mathcal{c}}(\mathrm{x})\right|\left(\nabla\left|\varphi_{m}^{\mathcal{C}}(\mathbf{x})\right\rangle\right)=\left\langle\varphi_{0}(\mathrm{x})\left|U^{-1}(\hat{x})\left(\nabla_{\hat{x}} U(\hat{x})\right)\right| \varphi_{m}(\mathrm{x})\right\rangle+\left\langle\varphi_{0}(\mathrm{x})\right|\left(\nabla_{\mathbf{x}}\left|\varphi_{m}(\mathrm{x})\right\rangle\right)
$$

The first term on the right hand side of Eq. 21 is not necessarily small. The second term is the non-adiabatic coupling matrix..$^{3,21,31,32} \mathrm{By}$ differentiating the electronic eigenvalue equation of Eq. 3, multiplying on the left with $\varphi(\mathbf{r} ; \mathbf{x})$ and integrating over $\mathbf{r}$, we find that $(\text { for } m \neq 0)^{3,21}$

$$
\left\langle\varphi_{0}(\mathrm{x})\right|\left(\nabla_{\mathrm{x}}\left|\varphi_{m}(\mathrm{x})\right\rangle\right)=\frac{\left\langle\varphi_{0}(\mathrm{x})\left|\left(\nabla_{\mathrm{x}} h(\mathrm{x})\right)\right| \varphi_{m}(\mathrm{x})\right\rangle}{V_{m}(\mathrm{x})-V_{0}(\mathrm{x})}
$$

For low scattering and bound state energies (which we are considering), the denominator in Eq. 22 is always much larger than the numerator. Then, the second term on the right hand side of Eq. 21 is small and can be neglected.

For the molecules and energies which we are considering, the electronic angular momentum is typically smaller than the other angular momenta in the molecule. ${ }^{8}$ Thus, we neglect the terms which involve the electronic angular momentum: the $\mathbf{A}(\mathrm{x}, \hat{x})$ and the first term on the right hand side of Eq. 21. The relevant nuclear Schrödinger equation is then given by Eq. 12 with $\varepsilon(\mathbf{x})=V(\mathrm{x})$ and $\mathbf{A}(\mathrm{x})=\mathbf{A}(\mathrm{x})$.

\section{GEOMETRIC PHASE EFFECTS IN $\mathrm{H}+\mathrm{O}_{2}$ SCATTERING}

The computational techniques which are needed to solve the generalized BornOppenheimer equation of Eq. 12 have only recently been developed. ${ }^{8}$ A straightforward application of conventional techniques was not possible due to the singular behavior of the vector potential terms. Instead, a new hybrid approach was developed which utilized both a DVR (Discrete Variable Representation) and an FBR (Finite Basis Representation). ${ }^{8}$ By using very large quadratures in the FBR, the singular behavior of the vector potential terms could be integrated out and an accurate DVR representation obtained (via transformation 
from the FBR). The Sequential Truncation Diagonalization technique was implemented in order to reduce the size of the final DVR Hamiltonian to be diagonalized. ${ }^{33}$ Two main features of the hybrid method are: 1) It is highly parallelizable and is currently being implemented on the Cray $\mathrm{T}^{3} \mathrm{D}^{34}$ and 2) It solves the complex Hermitian eigenvalue problem of Eq. 12 by efficient diagonalization of a real symmetric matrix of the same dimension. A detailed description of this technique can be found in Ref. 8 .

The newly developed hybrid technique has recently been applied to low energy (nonreactive) $\mathrm{H}+\mathrm{O}_{2}$ scattering and $\mathrm{HO}_{2}$ bound state calculations for zero total angular momentum $(J=0) .^{9,11}$ These calculations were the first to include geometric phase effects in a real molecule using the vector potential (gauge theory) approach. In this section we discuss the symmetry effects on the rovibrational wavefunctions due to the pseudo magnetic field (i.e. the geometric phase) which originates from the $C_{2 v}$ conical intersection in $\mathrm{HO}_{2}$. The effects on the resonance spectrum and the $\mathrm{O}_{2}$ vibrational relaxation probability are also presented.

A 2D slice of the 3D potential energy surface (PES) for the ground $\left({ }^{2} A^{\prime \prime}\right)$ electronic state of $\mathrm{HO}_{2}$ is plotted in Fig. 2. This DIM (Diatomics In Molecules) surface is the newest, most accurate global representation to date and is based on approximately 900 very accurate $a b$ initio points. ${ }^{35-38}$ It is a double-valued surface which gives true conical intersections. The $\mathrm{O}_{2}$ bond is fixed at $2.5 \mathrm{a}_{0}$ and the $R_{x}$ and $R_{y}$ coordinates denote the position of the $\mathrm{H}$ atom relative to the center of the $\mathrm{O}_{2}$ bond. The two deep attractive wells are clearly visible which represent the $\mathrm{H}$ atom being bound to one or the other $\mathrm{O}$ atoms. The lower cone of the $C_{2 v}$ conical intersection is clearly visible along the $R_{y}$ axis with $R_{x}=0$ (T-shaped geometries). The two halves of the lower cone for the linear conical intersection are also visible along the $R_{x}$ axis with $R_{y}=0$ (linear geometries). The minimum energy pathway around the $C_{2 v}$ conical intersection is always below the dissociation energy. Thus, even for scattering energies just above threshold, nuclear motion around the $C_{2 v}$ conical intersection is classically allowed and we expect significant geometric phase effects. The minimum energy for the linear saddle point is $0.42 \mathrm{eV} .{ }^{35}$ Thus, if we restrict our scattering energies to below 
$0.40 \mathrm{eV}$, nuclear motion which encircles the linear conical intersection is classically forbidden and we can neglect the geometric phase effects originating from this intersection.

The real electronic wavefunction is double-valued around the $C_{2 v}$ conical intersection. This double-valuedness gives rise to unusual symmetry behavior of the electronic wavefunction under a permutation $(\mathcal{P})$ of the two identical ${ }^{16} \mathrm{O}$ atoms. ${ }^{8,10}$ The real electronic wavefunction is simultaneously antisymmetric in front of the conical intersection (in the dissociation region) and symmetric behind the conical intersection (across the $C_{2 v}$ saddle region). The nuclear spin wavefunction is always symmetric since ${ }^{16} \mathrm{O}$ has zero nuclear spin. Thus, in order for the total wavefunction to satisfy Bose statistics $\left({ }^{16} \mathrm{O}\right.$ is a spin 0 Boson) for all nuclear geometries, the nuclear wavefunction must also be double-valued and exhibit the same unusual permutation behavior.

As discussed in the previous section, the correct double-valued behavior of the real nuclear wavefunctions is obtained by solving the generalized Born-Oppenheimer equation of Eq. 12. The appropriate phase factor and projection operator is applied to the complex solutions to obtain real double-valued solutions in the following way ${ }^{8,11}$

$$
\Psi_{+}=\sigma^{+} \exp \left[i \frac{l}{2} \eta\right] \Psi^{\mathcal{C}} \quad, \quad \Psi_{-}=-i \sigma^{-} \exp \left[i \frac{l}{2} \eta\right] \Psi^{\mathcal{c}}
$$

where $\sigma^{ \pm} \equiv(1 \pm \mathcal{P}) / 2$ and the + and - designate symmetric or antisymmetric asymptotic symmetry (i.e. in front of the conical intersection). As discussed above, the nuclear spin of ${ }^{16} \mathrm{O}$ is zero and the electronic wavefunction is antisymmetric asymptotically, so that only the $\Psi_{-}$are physically allowed. The qualitative behavior of the $\Psi_{-}$is shown in Fig. 3 where a 1D slice (solid curve) of the 3D real double-valued solution is overlaid on top of a stereographic projection of the $\mathrm{HO}_{2}$ PES in hyperspherical coordinates. ${ }^{8}$ The $C_{2 v}$ conical intersection is clearly visible in the center of the plot as are the two deep attractive wells on either side. The repulsive potential for $x>0$ and the tops of the $C_{2 v}$ and linear conical intersections are cut-off for clarity. The curve is clearly double-valued and is simultaneously antisymmetric in front of the $C_{2 v}$ conical intersection and symmetric behind. The wavefunction changes sign for one complete $2 \pi$ revolution around the intersection. Encircling the conical intersection 
twice brings the wavefunction back to its initial starting point.

The real double-valued nuclear wavefunctions are used to compute the scattering matrix S. ${ }^{8,14}$ By computing the scattering matrix and its derivative at many different scattering energies, a collision lifetime matrix $\mathbf{Q}$ can be computed as a function of energy. ${ }^{39}$ The trace of $\mathbf{Q}$ (i.e. $\operatorname{Tr} \mathbf{Q}(E)$ ) gives the resonance spectrum which is plotted in Fig. 4. The "spikes" are quantum resonances where the $\mathrm{H}$ atom has collided with the $\mathrm{O}_{2}$ diatom to form a short lived complex (resonance state). Many narrow (long-lived) and broad (short-lived) resonances are clearly visible. The upper plot is computed with No Geometric Phase (NGP) using the standard Born-Oppenheimer equation of Eq. 6 (i.e. $l=0$ ). The lower plot is computed with the Geometric Phase (GP) using the generalized Born-Oppenheimer equation of Eq. 12 with $l=1$. Significant differences between the two are clearly visible. Results for the entire energy range between threshold $(0.0978 \mathrm{eV})$ and $0.40 \mathrm{eV}$ are presented in Ref. 11 .

The scattering matrix is also used to compute state-to-state transition probabilities. Figure 5 plots the $\left(v=0, \bar{j}_{i}\right) \rightarrow\left(v=1, \sum j_{f}\right)$ vibrational relaxation probability as a function of energy for $E \geq 0.29 \mathrm{eV}$ (the $\mathrm{O}_{2}(v=1)$ channel opens up at $0.291 \mathrm{eV}$ ). The symbol $\bar{j}_{i}$ indicates that the open initial rotational quantum numbers of $\mathrm{O}_{2}$ have been Boltzmann averaged. That is, all of the transition probabilities $\left(v=0, j_{i}\right) \rightarrow\left(v=1, \sum j_{f}\right)$ for each open $j_{i}$ are weighted by the appropriate Boltzmann factor and added together. The $\sum j_{f}$ indicates that all final open rotational quantum numbers have been summed over. The NGP results for the vibrational relaxation probability show much more oscillatory behavior than the GP results. An underlying "stair-step" structure can be seen in both the NGP and GP results by averaging out the oscillations (see the solid horizontal lines in Fig. 5). The slope of the "staircase" (i.e. the average rate of increase of this transition probability with energy) is approximately the same for both the NGP and GP results. However, the "staircase" (i.e. the average transition probability) for the GP results is shifted lower in probability $(\approx 0.025$ lower) than the NGP results. Thus, the "cumulative transition probability" for the lowest vibrational transition in $\mathrm{H}+\mathrm{O}_{2}$ (nonreactive) scattering for $J=0$ is significantly attenuated $(\approx 35 \%)$ by the geometric phase. The results for several other state-to-state 
transition probabilities are presented in Ref. 11.

The low energy $\mathrm{H}+\mathrm{O}_{2}$ scattering results clearly indicate that the effects of a pseudo magnetic field (i.e. the geometric phase) are real and must be included in order to obtain the correct results. Work is currently underway to extend the scattering calculations to non-zero $J .^{34}$ The results of these calculations will eventually allow for a direct comparison with $\mathrm{H}$ $+\mathrm{O}_{2}$ scattering experiments. Based on the large differences seen in the $J=0$ results, we fully expect that the inclusion of geometric phase effects will be necessary in order to obtain agreement between theory and experiment.

\section{GEOMETRIC PHASE EFFECTS IN THE VIBRATIONAL SPECTRA OF $\mathrm{NA}_{3}(\mathrm{X})$}

The alkali metal trimer $\mathrm{Na}_{3}$ has been under intense theoretical ${ }^{40-49}$ and experimental ${ }^{50-57}$ investigation in recent years. Almost all interest has been focused on the nuclear dynamics in the excited $B$ electronic state. In fact, the vibrational spectra of $\mathrm{Na}_{3}(\mathrm{~B})$ was originally believed to be the first example of the geometric phase effect in molecular spectra. ${ }^{51}$ However, in recent years a more detailed theoretical treatment ${ }^{45-47}$ together with higher resolution spectroscopy ${ }^{55}$ have reversed this original claim. It is now clear that there is no geometric phase effect in the vibrational spectra for the $B$ electronic state. The $B$ state is of ${ }^{2} A_{1}^{\prime}$ symmetry (instead of ${ }^{2} E^{\prime}$ as was once thought) and exhibits an avoided crossing at the $D_{3 h}$ geometry with the nearby ${ }^{2} E^{\prime}$ doubly degenerate state. Thus, the vibrational wavefunctions are single-valued and the spectra can be classified using a pseudo-Jahn-Teller (PJT) coupling scheme. ${ }^{45-47}$

Interestingly, the nuclear dynamics on the ground electronic state $\left(\mathrm{Na}_{3}(\mathrm{X})\right)$ has received very little attention (both theoretical and experimental) even though it is well known that a conical intersection is located at the equilateral triangular configuration ( $D_{3 h}$ symmetry). ${ }^{40}$ The pseudorotational barrier is much lower in energy than the conical intersection and the wells between the barriers are relatively shallow. Thus, even low vibrational states 
could exhibit significant geometric phase effects. Calculations which include geometric phase effects in the vibrational dynamics have been performed for other alakli metal trimers such as $\mathrm{Li}_{3}{ }^{50,42,58}$ and $\mathrm{Cu}_{3}{ }^{42,59}$ using a two-state diabatic representation. The conical intersection between the ground and first excited electronic states in these two molecules lies very low in energy. Only the lowest few vibrational energies lie below the energy of the low-lying conical intersection. Thus, an adiabatic (single electronic state) approximation is not valid for these molecules and a coupled two-state calculation is required. While a careful two-state calculation can certainly include geometric phase effects, the additional large non-adiabatic effects in $\mathrm{Li}_{3}$ and $\mathrm{Cu}_{3}$ make it difficult to determine which effects are due to the geometric phase. Until now, there has been no "clean" (i.e. dynamics on a single adiabatic ground state electronic PES with a conical intersection) example of a geometric phase effect in molecular spectra.

The first calculations to include the geometric phase in the vibrational dynamics of a molecule using a single adiabatic ground state electronic PES have only recently been performed. ${ }^{11}$ In these calculations, all 249 bound states of $\mathrm{HO}_{2}$ were computed up to the dissociation energy. However, even though there is a minimum energy pathway around the $C_{2 v}$ intersection which lies below the dissociation energy, the PES is such that even the highest bound states are localized over the deep attractive wells and have zero amplitude in front of the intersection. Thus, they do not "feel" the effects of the geometric phase and no shifts in the energy levels between the GP calculations and the NGP calculations using an even (single-valued) basis set were observed. However, it is important to realize that the correct physical states include the geometric phase and are therefore even across the $C_{2 v}$ saddle region instead of odd ${ }^{8,10,60}$ Several recent calculations which were unaware of this symmetry effect have computed the bound states using an odd (single-valued) basis set. ${ }^{61-64}$ There is significant splitting (up to $100 \mathrm{~cm}^{-1}$ ) between the energy levels of even and odd symmetry which lie above the minimum $C_{2 v}$ saddle point energy. ${ }^{11}$

A contour plot of the PES for $\mathrm{Na}_{3}(\mathrm{X})$ for a fixed value of the hyperradius $\rho=8.71 \mathrm{a}_{0}$ is shown in Fig. 6. This surface was fit by Thompson, et al. ${ }^{43}$ to the ab initio data of 
Martins, et $a .^{41}$ The value of $\rho=8.71 \mathrm{a}_{0}$ is chosen since it gives the minimum energy in the wells. Motion along the hyperradius $\rho$ corresponds to the symmetric stretch mode $\left(Q_{s}\right)$, motion along the $x$ axis corresponds to the bending mode $\left(Q_{x}\right)$, and motion along the $y$ axis corresponds to the antisymmetric stretch mode $\left(Q_{y}\right)$. A conical intersection occurs at the origin $(x=0, y=0)$ which corresponds to the equilateral triangular configuration $\left(D_{3 h}\right.$ symmetry). The three fold symmetry gives rise to three shallow wells which are equally spaced at $120^{\circ}$ intervals around the origin. The minimum energies relative to the bottom of the wells for the pseudorotational barrier (i.e. the saddle points between the three wells) and the conical intersection are $292.6 \mathrm{~cm}^{-1}$ and $962.1 \mathrm{~cm}^{-1}$, respectively. Thus, the conical intersection in $\mathrm{Na}_{3}(\mathrm{X})$ lies high enough in energy so that for low-lying vibrational states the adiabatic approximation is valid and the generalized Born-Oppenheimer method of Section III can be applied to include geometric phase effects in the vibrational dynamics.

Unlike the zero spin ${ }^{16} \mathrm{O}$ atoms in $\mathrm{HO}_{2}$, the ${ }^{23} \mathrm{Na}$ atoms have non-zero nuclear spin $(S=$ $3 / 2$ ) and are Fermions. Thus, the determination of the physically allowed products of the vibrational, electronic, and nuclear spin wavefunctions is slightly more complicated than for $\mathrm{HO}_{2}$. The total number of nuclear spin states is $(2 S+1)^{3}=64$ which can be classified using the irreducible representations of the permutation group $S_{3}$. The irreducible representations of $S_{3}$ are: $A_{1}$ (symmetric), $A_{2}$ (antisymmetric), and $E$ (doubly degenerate). The number of nuclear spin states of $A_{1}, A_{2}$, and $E$ symmetry are given by: $(2 S+1)(2 S+3)(S+1) / 3=20$, $(2 S+1)(2 S-1) S / 3=4$, and $(2 S+1)(S+1) 8 S / 3=40$, respectively. ${ }^{65}$ The real electronic wavefunction is double-valued around the conical intersection (origin) and simultaneously exhibits $A_{1}$ symmetry across the three saddle points and $A_{2}$ symmetry across the centers of the three wells. ${ }^{40}$ We will first consider only symmetric nuclear spin functions. Then, in order for the total wavefunction to satisfy Fermi statistics for all nuclear geometries, the vibrational wavefunction must also be double-valued but exhibit the opposite symmetry behavior. That is, it must be of $A_{2}$ symmetry across the saddle points and of $A_{1}$ symmetry across the wells. The qualitative behavior of this real double-valued vibrational wavefunction is shown in Fig. 7 which plots a $1 \mathrm{D}$ slice of the $3 \mathrm{D}$ wavefunction around the conical intersection. For 
the antisymmetric nuclear spin states the symmetry is reversed. That is, a real doublevalued vibrational wavefunction must be of $A_{1}$ symmetry across the saddle points and of $A_{2}$ symmetry across the wells. The qualitative behavior of this wavefunction is plotted in Fig. 8. The nuclear spin states of $E$ symmetry can only be combined with doublevalued vibrational states of $E$ symmetry to produce a direct product representation which simultaneously exhibits $A_{2}$ symmetry across the saddle points and $A_{1}$ symmetry across the centers of the wells. ${ }^{6}$ Calculations of the double-valued vibrational wavefunctions of $E$ symmetry and the corresponding energy spectrum are currently underway and will be presented in future work. ${ }^{67}$

The bound state techniques which were used to include geometric phase effects in the vibrational spectrum of $\mathrm{HO}_{2}$ were modified to handle three identical nuclei and applied to $\mathrm{Na}_{3}{ }^{11,67}$ The relevant phase factors and projection operators for the $A_{1}$ and $A_{2}$ irreducible representations of $S_{3}$ are applied to the complex solutions $\Psi^{\mathcal{C}}$ to obtain real double-valued solutions $\Psi$ with the correct $A_{1}$ or $A_{2}$ symmetry in the following way ${ }^{3}$

$$
\Psi=\left[\left(1+\mathcal{P}_{123}+\mathcal{P}_{321}\right) \Psi^{\mathcal{C}}\right] e^{i \frac{l}{2} \eta} \pm\left[\left(\mathcal{P}_{12}+\mathcal{P}_{23}+\mathcal{P}_{31}\right) \Psi^{\mathcal{C}}\right] e^{-i \frac{l}{2} \eta}
$$

where the $+\operatorname{sign}$ gives $A_{2}$ symmetry and the $-\operatorname{sign} A_{1}$ symmetry (the symmetry designation refers to the symmetry behavior across the wells). For $X_{3}$ systems the most convenient choice of odd integer $l$ for the treatment of permutation symmetry is $l=3 .^{3}$

The results of the $3 \mathrm{D}$ bound state calculations for the $A_{1}$ and $A_{2}$ vibrational energy levels of $\mathrm{Na}_{3}(\mathrm{X})$ are presented in Fig. 9. The energies for the lowest 25 states of both symmetries were computed with the geometric phase (GP) using $l=3$ and without (NGP) by setting $l=0$. Since there are 5 times more $A_{1}$ nuclear spin states than $A_{2}$, the $A_{1}$ vibrational spectrum will be on average 5 times more intense than the $A_{2}$ spectrum. Thus, the $A_{1}$ energy levels are presented using solid horizontal lines while the $A_{2}$ energy levels are denoted by dashed horizontal lines. All energies are relative to the bottom of the $\mathrm{Na}_{3}$ wells. The long horizontal dotted lines labeled by SP and CI denote the minimum saddle point energy and the minimum conical intersection energy, respectively. Only the lowest (ground state) energy 
of $A_{1}$ symmetry lies below the pseudorotational barrier (the minimum saddle point energy). The other low-lying states of $A_{1}$ and $A_{2}$ symmetry lie above the pseudorotational barrier but their wavefunctions remain localized over the attractive wells and no energy differences between the GP and NGP results are observed. However, for the intermediate to high-lying states, we see significant differences between the GP and NGP results for both symmetries. The $A_{1}$ GP energy levels are more closely spaced at high energies when compared to the $A_{1}$ NGP spectrum while the $A_{2}$ GP energy levels are less closely spaced at high energies when compared to the $A_{2}$ NGP spectrum.

Table I gives the precise energy values presented in Fig. 9. Detailed convergence studies indicate that the relative accuracy for the lowest ten energy levels is $<0.25 \mathrm{~cm}^{-1}$ while for the higher levels it is $<1.0 \mathrm{~cm}^{-1}$. ${ }^{67}$ The absolute accuracy is estimated to be $<1.0 \mathrm{~cm}^{-1}$ for the lowest ten energy levels and $<2.0 \mathrm{~cm}^{-1}$ for the higher ones. In comparing the GP and NGP results in Table I, it is the relative accuracy which is important. Thus, any differences between the GP and NGP energies for the low-lying states greater than $0.5 \mathrm{~cm}^{-1}$ are real and due entirely to the geometric phase. We see that the GP and NGP energies for the lowest two states of $A_{1}$ symmetry and the lowest three states of $A_{2}$ symmetry are identical (within convergence errors). Significant splitting begins to appear in the third state of $A_{1}$ symmetry and the fourth state of $A_{2}$ symmetry. For higher energies the wavefunctions become less localized over the wells and the energy differences between the GP and NGP results for both symmetries increase. The high-lying wavefunctions have significant amplitude all the way around the conical intersection and therefore exhibit large geometric phase effects.

The bound state results for the $A_{1}$ and $A_{2}$ vibrational spectrum of $\mathrm{Na}_{3}(\mathrm{X})$ clearly show that geometric phase effects must be included in order to obtain the correct results. The vibrational state assignments (i.e. the assignment of the symmetric stretch, bending, and asymmetric stretch quantum numbers) for the $A_{1}$ and $A_{2}$ energy levels and the calculations of the degenerate $E$ spectrum and state assignments are currently underway. ${ }^{67}$ The results of these calculations together with the present results will provide a detailed theoretical understanding of the $\mathrm{Na}_{3}(\mathrm{X})$ vibrational spectrum (for $J=0$ ) including geometric phase 
effects. Unfortunately, direct comparison with experiment is not yet possible due to the lack of experimental data and the limited accuracy of the PES for $\mathrm{Na}_{3}(\mathrm{X})$. The $\mathrm{Na}_{3}(\mathrm{X})$ PES is based on relatively old $a b$ initio data ${ }^{41}$ which could be improved by using more modern electronic structure techniques. The only experimental results to date for the vibrational

levels of $\mathrm{Na}_{3}(\mathrm{X})$ are based on two-photon ionization spectra of the hot bands. ${ }^{52}$ Only the three fundamentals were reported. It has recently been proposed that stimulated emission pumping or IR excitation could be used to measure the vibrational spectra of $\mathrm{Na}_{3}(\mathrm{X})$ and investigate geometric phase effects. ${ }^{48}$ Future theoretical work (i.e. the development of an improved PES for $\mathrm{Na}_{3}(\mathrm{X})$ ) and new experimental data for many intermediate to high-lying vibrational states should provide a "clean" example of the geometric phase effect in molecular spectra.

\section{CONCLUSIONS}

We have seen that pseudo magnetic fields appear in the Born-Oppenheimer method when conical intersections or electronic angular momenta are taken into account. The pseudo magnetic field which originates from a conical intersection is that of a "magnetic solenoid". The "magnetic solenoid" gives rise to a geometric (Berry) phase which can lead to real physical effects in the molecular dynamics. The results of low-energy $\mathrm{H}+\mathrm{O}_{2}$ scattering calculations for $J=0$ showed significant shifts in the resonance energies and lifetimes due to the geometric phase associated with the $C_{2 v}$ conical intersection in $\mathrm{HO}_{2}$. A significant attenuation of the $\mathrm{O}_{2}$ vibrational relaxation probability was also observed. The first calculations to include geometric phase effects in the vibrational spectrum of $\mathrm{Na}_{3}(\mathrm{X})$ were presented. Significant shifts in the intermediate to high-lying energy levels of $A_{1}$ and $A_{2}$ symmetry were observed due the geometric phase associated with the $D_{3 h}$ conical intersection. The results of these calculations clearly show that the geometric phase is important and in many cases it must be included in order to obtain the correct results and agreement between theory and experiment. 
The pseudo magnetic fields and their associated geometric phase exist in the absence of real external magnetic fields. However, recent ab initio calculations have shown that real magnetic fields can induce conical intersections in the excited electronic energy levels of the $\mathrm{H}_{2}^{+}$ion. ${ }^{18-20}$ Conical intersections are normally not allowed in diatomic molecules due to the fact that there is only one internal degree of freedom (the internuclear distance). However, in the presence of an external magnetic field, the orientation of the magnetic field with respect to the internuclear axis of the diatom introduces an additional degree of freedom (i.e. the PES is 2D instead of 1D) so that conical intersections can exist. These results suggest an interesting experiment where the rovibrational spectroscopy of an excited electronic state of a molecule is measured in the presence of a magnetic field for varying field strengths. If a conical intersection is created or destroyed at some critical value of the field strength, then a sudden (discontinuous) jump in the rovibrational energy levels would be observed. The sudden jump in the energy levels would be a direct consequence of the geometric phase which suddenly "turns on" when the conical intersection is created or "turns off" when it is destroyed. This approach would give experimentalist the ability to "turn on" or "turn off" the geometric phase at will and measure the differences in the spectra. Unfortunately, the field strengths required to significantly alter the low-lying electronic energy levels are quite large $\left(10^{5} \mathrm{~T}\right)$ and are far beyond the current capabilities of continuous field laboratory magnets $(30 \mathrm{~T})$. The situation may be more promising for high-lying electronic states but then an accurate theoretical treatment becomes more difficult. The huge magnetic fields found on the surfaces of astrophysical objects such as white dwarfs $\left(10^{2}-10^{5} \mathrm{~T}\right)$ and neutron stars $\left(10^{7}-10^{9} \mathrm{~T}\right)$ are certainly large enough to induce conical intersections in molecules. Thus, geometric phase effects could contribute to the unusual behavior of the rovibrational spectra of the molecules found in the atmospheres of these objects.

Another interesting effect due to real magnetic fields is the coupling of the center of mass (CM) motion to the internal degrees of freedom. ${ }^{15}$ Thus, the CM motion can induce transitions between different rovibrational and/or electronic states of a molecule which is moving in the presence of a magnetic field. At the same time, magnetic fields also increase the 
binding energies in molecules which leads to deeper minima in the PES. ${ }^{17-20}$ For the $\mathrm{H}_{2}^{+}$ion for example, one of the rotational degrees of freedom acquires a vibrational character which becomes more pronounced with increasing field strength. ${ }^{19}$ The resulting dynamics is that of hindered rotation. Calculations of the rovibrational spectra of the $\mathrm{H}_{2}^{+}$ion could be performed including the CM coupling effects. The CM coupling is proportional to the field strength and CM velocity. ${ }^{15}$ Thus, the transition probabilities between the rovibrational states could be computed as a function of the field strength and the CM velocity. Comparison with laboratory experiments might be possible since high beam energies (large $\mathrm{CM}$ velocities) could be used to magnify the coupling effects (if needed).

The development of the necessary theoretical and computational techniques for including the effects of real magnetic fields in molecular dynamics is far from complete. Recent methods which use gauge theory techniques for treating pseudo magnetic fields should help in the development of methods for including the effects of real magnetic fields. ${ }^{8}$ As discussed above, effects due to both types of fields can be present at the same time. Since the two types of fields are described formally by replacing the momentum operator $\mathbf{p}$ by $\mathbf{p}-\mathbf{A}$, a general gauge theory approach which includes the effects of both types of fields would be based on the replacement $\mathbf{p} \rightarrow \mathbf{p}-\left(\mathbf{A}_{\text {pseudo }}+\mathbf{A}_{\text {real }}\right)$ where $\mathbf{A}_{\text {pseudo }}$ and $\mathbf{A}_{\text {real }}$ are the relevant vector potentials for the pseudo magnetic field and real magnetic field, respectively. Much more theoretical work is needed to develop accurate and efficient computational methods for including the effects of magnetic fields in molecules. The study of magnetic field effects in molecular dynamics is certainly an exciting area of research which should provide many new theoretical and experimental discoveries. 


\section{REFERENCES}

${ }^{1}$ For a review, see: R. T Pack and J. O. Hirschfelder, J. Chem. Phys. 49, 4009 (1968); ibid. 52521 (1970); ibid. 4198 (1970).

${ }^{2}$ G. Herzberg and H. C. Longuet-Higgins, Discuss. Faraday Soc. 35, 77 (1963).

${ }^{3}$ C. A. Mead and D. G. Truhlar, J. Chem. Phys. 70, 2284 (1979); C. A. Mead, J. Chem. Phys. 72, 3839 (1980).

${ }^{4}$ C. A. Mead, Chem. Phys. 49, 23 (1980).

${ }^{5}$ M. V. Berry, Proc. R. Soc. London, Ser. A 392, 45 (1984); in Geometric Phases in Physics, edited by A. Shapere and F. Wilczek, (World Scientific, Singapore, 1989); Phys. Today 43 (12), 34 (1990).

${ }^{6}$ A. Kuppermann, in Dynamics of Molecules and Chemical Reactions, edited by R. E. Wyatt and J. Z. H. Zhang, (Marcel Dekker, Inc., New York, 1996), and references therein.

${ }^{7}$ X. Wu, R. E. Wyatt, and M. D'Mello, J. Chem. Phys. 101, 2953 (1994).

${ }^{8}$ B. Kendrick and R. T Pack, J. Chem. Phys. 104, 7475 (1996), and references therein.

${ }^{9}$ B. Kendrick and R. T Pack, J. Chem. Phys. 104, 7502 (1996).

${ }^{10}$ V. J. Barclay, C. E. Dateo, I. P. Hamilton, B. Kendrick, R. T Pack and D. W. Schwenke, J. Chem. Phys. 103, 3864 (1995).

${ }^{11}$ B. Kendrick and R. T Pack, J. Chem. Phys., (in press).

${ }^{12}$ F. T. Smith, J. Math. Phys. 3, 735 (1962).

${ }^{13}$ B. R. Johnson, J. Chem. Phys. 73, 5051 (1980); J. Chem. Phys. 79, 1906 (1983); ibid. 1916 (1983).

${ }^{14}$ R. T Pack and G. A. Parker, J. Chem. Phys. 87, 3888, (1987).

${ }^{15}$ P. Schmelcher, L. S. Cederbaum, and H.-D. Meyer, Phys. Rev. A 38, 6066 (1988). 
${ }^{16}$ P. Schmelcher, L. S. Cederbaum, and U. Kappes, in Conceptual Trends in Quantum Chemistry, (Kluwer Academic Publisher, 1994) pp. 1-51.

${ }^{17}$ U. Kappes and P. Schmelcher, J. Chem. Phys. 100, 2878 (1994), and references therein.

${ }^{18}$ U. Kappes and P. Schmelcher, Phys. Rev. A 51, 4542 (1995); ibid. 53, 3869 (1996); ibid. 54, 1313 (1996).

${ }^{19}$ P. Schmelcher and L. S. Cederbaum, Phys. Rev. A 41, 4936 (1990).

${ }^{20}$ U. Kappes and P. Schmelcher, Phys. Lett. A 210, 409 (1996).

${ }^{21}$ C. A. Mead, Rev. Mod. Phys. 64, 51 (1992).

${ }^{22}$ B. Kendrick and C. A. Mead, J. Chem. Phys. 102, 4160 (1995).

${ }^{23}$ The global nature of the sign change can be understood topologically by realizing that within the adiabatic approximation the degeneracy manifold $\mathcal{D}$ effectively "punches a hole" in $\mathcal{M}$ so that the effective nuclear parameter space $\mathcal{M}-\mathcal{D}$ is not simply connected. Thus, a vector potential of the form $\mathbf{A}=(-l / 2) \nabla_{x} \eta(\mathrm{x})$ (l odd integer) is not a pure gauge and therefore gives rise to a geometric phase which can lead to real physical effects in the molecular dynamics.

${ }^{24}$ The full $2 \pi$ range for $\eta$ is determined from the signs of the $v(\mathrm{x})$ and $u(\mathrm{x})$ functions which appear in the $\tan ^{-1}$ function.

${ }^{25}$ M. E. Rose, Elementary Theory of Angular Momentum (Wiley, New York, 1957).

${ }^{26}$ A. S. Davydov, Quantum Mechanics, (Pergamon Press, New York, 1985), 2nd edition.

${ }^{27}$ A. Bohm, B. Kendrick, and M. Loewe, Int. J. Quantum Chem. 41, 53 (1992); A. Bohm, B. Kendrick, M. Loewe, and L. J. Boya, J. Math. Phys. 33, 977 (1992).

${ }^{28}$ J. Moody, A. Shapere, and F. Wilczek, Phys. Rev. Lett. 56, 893 (1986).

${ }^{29}$ R. Jackiw, Phys. Rev. Lett. 56, 2779 (1986). 
${ }^{30}$ B. Zygelmann, Phys. Rev. Lett. 64, 256 (1990); Phys. Lett. A 125, 476 (1987).

${ }^{31}$ C. A Mead and D. G. Truhlar, J. Chem. Phys. 77, 6090 (1982).

${ }^{32}$ C. A Mead, J. Chem. Phys. 78, 807 (1983).

${ }^{33}$ R. M. Whitnell and J. C. Light, J. Chem. Phys. 90, 1774 (1989), and references therein.

${ }^{34}$ B. Kendrick and E. F. Hayes, (unpublished).

${ }^{35}$ B. Kendrick and R. T Pack, J. Chem. Phys. 102, 1994 (1995).

${ }^{36}$ S. P. Walch, C. M. Rohlfing, C. F. Melius, and C. W. Bauschlicher, Jr., J. Chem. Phys. 88, 6273 (1988); 90, 7613 (1989).

${ }^{37}$ S. P. Walch and C. M. Rohlfing, J. Chem. Phys. 91, 2373 (1989).

${ }^{38}$ S. P. Walch and R. J. Duchovic, J. Chem. Phys. 94, 7068 (1991); 96, 4050 (1992).

${ }^{39}$ F. T. Smith, Phys. Rev. 118, 349 (1960).

${ }^{40}$ R. L. Martin and E. R. Davidson, Molec. Phys. 35, 1713 (1978).

${ }^{41}$ J. L. Martins, R. Car, and J. Buttet, J. Chem. Phys. 78, 5646 (1983).

${ }^{42}$ T. C. Thompson, D. G. Truhlar, and C. A. Mead, J. Chem. Phys. 82, 2392 (1985).

${ }^{43}$ T. C. Thompson, G. Izmirlian, Jr., S. J. Lemon, D. G. Truhlar, and C. A. Mead, J. Chem. Phys. 82, 5597 (1985).

${ }^{44}$ F. Cocchini, T. H. Upton, and W. Andreoni, J. Chem. Phys. 88, 6068 (1988).

${ }^{45}$ R. Meiswinkel and H. Köppel, Chem. Phys. 144, 117 (1990).

${ }^{46}$ J. Schön and H. Köppel, Chem. Phys. Lett. 231, 55 (1994)

${ }^{47}$ J. Schön and H. Köppel, J. Chem. Phys. 103, 9292 (1995).

${ }^{48}$ B. Reischl, Chem. Phys. Lett. 239, 173 (1995). 
${ }^{49}$ P.-H. Zhang and J.-M. Li, Phys. Rev. A 54, 665 (1996).

${ }^{50}$ A. Herrmann, M. Hofmann, S. Leutwyler, E. Schumacher, and L. Wöste, Chem. Phys. Lett. 62, 216 (1979).

${ }^{51}$ G. Delacrétaz, E. R. Grant, R. L. Whetten, L. Wöste, and J. W. Zwanziger, Phys. Rev. Lett. 56, 2598 (1986).

${ }^{52}$ M. Broyer, G. Delacrétaz, P. Labastie, J. P. Wolf, and L. Wöste, J. Phys. Chem. 91, 2626 (1987).

${ }^{53}$ H.-A. Eckel, J.-M. Gress, J. Biele, and W. Demtröder, J. Chem. Phys. 98, 135 (1993).

${ }^{54}$ J. Gaus, K. Kobe, V. Bonačić-Koutecký, H. Kühling, J. Manz, B. Reischl, S. Rutz, E. Schreiber, and L. Wöste, J. Phys. Chem. 97, 12509 (1993).

${ }^{55}$ W. E. Ernst and S. Rakowsky, Ber. Bunsenges. Phys. Chem. 99, 441 (1995).

${ }^{56}$ Th. Stoll, E. Tiemann, and D. Wendlandt, J. Molec. Spect. 174, 557 (1995).

${ }^{57}$ B. Reischl, R. de Vivie-Reidle, S. Rutz, and E. Schreiber, J. Chem. Phys. 104, 8857 (1996).

${ }^{58}$ M. Mayer and L. S. Cederbaum, J. Chem. Phys. 105, 4938 (1996).

${ }^{59}$ D. G. Truhlar, T. C. Thompson, and C. A. Mead, Chem. Phys. Lett. 127, 287 (1986).

${ }^{60}$ V. J. Barclay, C. E. Dateo, and I. P. Hamilton, J. Chem. Phys. 101, 6766 (1994).

${ }^{61}$ D. H. Zhang and J. Z. H. Zhang, J. Chem. Phys. 101, 3671 (1994).

${ }^{62}$ V. A. Mandelshtam, T. P. Grozdanov, and H. S. Taylor, J. Chem. Phys. 103, 10074 (1995).

${ }^{63}$ A. J. Dobbyn, M. Stumpf, H. M. Keller, W. L. Hase, and R. Schinke, J. Chem. Phys. 102, 5867 (1995); 103, 9947 (1995). 
${ }^{64}$ J. Dai and J. Z. H. Zhang, J. Chem. Phys., 104, 3664 (1996).

${ }^{65}$ D. M. Dennison, Rev. Mod. Phys. 3, 280 (1931).

${ }^{66}$ P. R. Bunker, Molecular Symmetry and Spectroscopy, (Academic Press, Inc., New York, 1979).

${ }^{67}$ B. Kendrick, (unpublished). 


\section{TABLES}

TABLE I. The lowest 25 bound state energies of $A_{1}$ and $A_{2}$ symmetry for $\mathrm{Na}_{3}(\mathrm{X})$ for zero total angular momentum $(J=0)$. The energies are in $\mathrm{cm}^{-1}$ and are relative to the bottom of the $\mathrm{Na}_{3}$ wells. The symmetry designations refer to the symmetry across the wells.

\begin{tabular}{|c|c|c|c|c|c|c|}
\hline State & $\operatorname{GP}\left(A_{1}\right)$ & $\operatorname{NGP}\left(A_{1}\right)$ & $\operatorname{diff}^{a}$ & $\operatorname{GP}\left(A_{2}\right)$ & $\operatorname{NGP}\left(A_{2}\right)$ & $\operatorname{diff}^{b}$ \\
\hline 0 & 238.5 & 238.6 & 0.1 & 341.4 & 341.3 & -0.1 \\
\hline 1 & 295.5 & 296.0 & 0.5 & 397.2 & 397.2 & 0.0 \\
\hline 2 & 349.3 & 350.8 & 1.5 & 449.5 & 449.8 & 0.3 \\
\hline 3 & 399.4 & 403.3 & 3.9 & 495.9 & 499.5 & 3.6 \\
\hline 4 & 439.0 & 438.9 & -0.1 & 531.6 & 534.4 & 2.8 \\
\hline 5 & 446.5 & 454.6 & 8.1 & 540.9 & 547.2 & 6.3 \\
\hline 6 & 487.6 & 489.1 & 1.5 & 578.1 & 586.4 & 8.3 \\
\hline 7 & 495.1 & 506.0 & 10.9 & 593.3 & 593.9 & 0.6 \\
\hline 8 & 528.3 & 531.6 & 3.3 & 626.4 & 632.6 & 6.2 \\
\hline 9 & 533.9 & 535.2 & 1.3 & 639.2 & 637.8 & -1.4 \\
\hline 10 & 546.1 & 558.8 & 12.7 & 644.4 & 643.3 & -1.1 \\
\hline 11 & 565.5 & 577.6 & 12.1 & 675.9 & 672.4 & -3.5 \\
\hline 12 & 587.6 & 592.2 & 4.6 & 695.4 & 690.5 & -4.9 \\
\hline 13 & 594.2 & 609.6 & 15.4 & 696.6 & 695.6 & -1.0 \\
\hline 14 & 601.0 & 624.0 & 23.0 & 713.5 & 708.0 & -5.5 \\
\hline 15 & 627.2 & 631.8 & 4.6 & 733.4 & 718.0 & -15.4 \\
\hline 16 & 628.3 & 649.0 & 20.7 & 749.8 & 739.7 & -10.1 \\
\hline 17 & 637.4 & 656.0 & 18.6 & 752.5 & 741.4 & -11.1 \\
\hline 18 & 646.5 & 674.8 & 28.3 & 761.2 & 747.1 & -14.1 \\
\hline 19 & 656.1 & 687.2 & 31.1 & 794.8 & 769.5 & -25.3 \\
\hline 20 & 679.5 & 688.7 & 9.2 & 799.5 & 775.1 & -24.4 \\
\hline 21 & 681.4 & 706.1 & 24.7 & 801.1 & 788.6 & -12.5 \\
\hline
\end{tabular}




\begin{tabular}{rllllll}
22 & 685.0 & 708.2 & 23.2 & 810.2 & 794.7 & -15.5 \\
23 & 694.0 & 721.5 & 27.5 & 816.8 & 796.1 & -20.7 \\
24 & 702.4 & 726.6 & 24.2 & 829.7 & 819.6 & -10.1 \\
\hline \hline
\end{tabular}

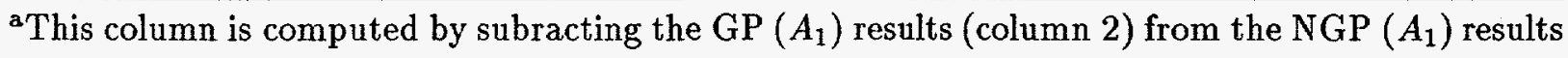
(column 3).

${ }^{\mathrm{b}}$ This column is computed by subtracting the GP $\left(A_{2}\right)$ results (column 5 ) from the NGP $\left(A_{2}\right)$ results (column 6). 


\section{FIGURES}

FIG. 1. Perspective plot of a 2D slice of a PES near a conical intersection. The degeneracy point is located at the origin in the $u v$ plane. The radial distance from the intersection is denoted by $r$ and the azimuthal angle around the intersection denoted by $\eta$. The adiabatic ground state electronic eigenvector changes sign for any closed path in $u v$ space which encircles the origin (such as the dashed curve $C$ ).

FIG. 2. Perspective plot of a $2 \mathrm{D}$ slice of the $3 \mathrm{D} \mathrm{HO}_{2}\left({ }^{2} A^{\prime \prime}\right)$ DIM PES. The $\mathrm{O}_{2}$ bond is fixed at $2.5 \mathrm{a}_{0}$. The coordinates $R_{x}$ and $R_{y}$ give the position of the $\mathrm{H}$ atom relative to the center of the $\mathrm{O}_{2}$ bond. The energy is relative to the bottom of the asymptotic $\mathrm{H}+\mathrm{O}_{2}$ channel. The deep attractive wells and the $C_{2 v}$ and linear conical intersections are clearly visible (see text for discussion).

FIG. 3. Perspective plot of the $\mathrm{HO}_{2}$ PES in hyperspherical coordinates with the hyperradius $\rho$ fixed at $4.9 \mathrm{a}_{0}$. The $x y$ coordinates are the stereographic projection of the surface of an upper half sphere. The energy is in $\mathrm{eV}$ and is relative to the bottom of the asymptotic $\mathrm{H}+\mathrm{O}_{2}$ well. The double-valued function $\sin (\eta / 2)$ is overlaid on top of the PES (dark curve). Extending $\eta$ over a $4 \pi$ range shows the full double-valued behavior by encircling the $C_{2 v}$ conical intersection twice. It is clear that the function is antisymmetric across the $x$ axis in front of the conical intersection and that it is symmetric across the $x$ axis behind the conical intersection. The double-valued function exhibits both symmetries simultaneously.

FIG. 4. Plot of $\operatorname{Tr} \mathbf{Q}^{0}$ as a function of energy: the points are calculated and the solid curves are a rational function interpolation. The energy is relative to the bottom of the $\mathrm{H}+\mathrm{O}_{2}$ channel. The upper plot was computed with No Geometric Phase (NGP) by setting $l=0$ and the lower plot was computed with the Geometric Phase (GP) using $l=1$. Significant differences are observed in the resonance energies and lifetimes (see text for discussion). 
FIG. 5. Plot of the $\left(v=0, \bar{j}_{i}\right) \rightarrow\left(v=1, \sum j_{f}\right) \mathrm{O}_{2}$ vibrational relaxation probability as a function of energy Boltzmann averaged over all open initial rotational quantum numbers and summed over all final rotational quantum numbers. Averaging out the oscillations gives rise to an underlying "stair step" structure (the solid horizontal lines). The GP results are significantly attenuated relative to the NGP results and are less oscillatory.

FIG. 6. Contour plot of the $\mathrm{Na}_{3}$ PES in hyperspherical coordinates with the hyperradius fixed at $\rho=8.71 \mathrm{a}_{0}$. This is a stereographic projection of the surface of an upper half sphere. The hyperangle $\theta$ runs from 0 at the north pole (center of plot) to $\frac{\pi}{2}$ at the equator (heavy circle). The hyperangle $\phi$, the azimuthal angle, is measured from the positive $x$-axis and goes to $\pi$ in the counterclockwise direction and to $-\pi$ in the clockwise direction. The contours are at $25,100,300$, $500,800,2000$, and $20000 \mathrm{~cm}^{-1}$ relative to the bottom of the $\mathrm{Na}_{3}$ wells. The highly repulsive region $\left(>20000 \mathrm{~cm}^{-1}\right)$ is shaded.

FIG. 7. Perspective plot of the $\mathrm{Na}_{3}$ PES in hyperspherical coordinates with the hyperradius fixed at $\rho=8.71 \mathrm{a}_{0}$. As in Fig. 6, the $x$ and $y$ coordinates are the stereographic projection of the surface of an upper half sphere. The energy is in $\mathrm{cm}^{-1}$ and relative to the bottom the $\mathrm{Na}_{3}$ wells. The repulsive region for negative values of $x$ and $y$ has been cut away in order to view the central region of the PES. The double-valued function $\sin (3 \eta / 2)\left(\right.$ for $\left.\mathrm{Na}_{3} \eta=\phi\right)$ is overlaid on top of the PES (dark curve). Extending $\eta$ over a $4 \pi$ range shows the full double-valued behavior by encircling the $D_{3 h}$ conical intersection twice. It is clear that this function is simultaneously symmetric across the three wells and antisymmetric across the three saddle points.

FIG. 8. Same plot as in Fig. 7 except that the double-valued function $\cos (3 \eta / 2)$ (for $\mathrm{Na}_{3}$ $\eta=\phi$ ) is overlaid on top of the PES (dark curve). It is clear that this function is simultaneously antisymmetric across the three wells and symmetric across the three saddle points. 
FIG. 9. The non-degenerate $A_{1}$ and $A_{2}$ vibrational spectra for $\mathrm{Na}_{3}(\mathrm{X})$ for zero total angular momentum $(J=0)$. The energies are in $\mathrm{cm}^{-1}$ and are relative to the bottom of the $\mathrm{Na}_{3}$ wells. The horizontal dotted lines labeled by SP and CI denote the minimum energies of the saddle points and the $D_{3 h}$ conical intersection, respectively. Due to the nuclear spin weighting, the $A_{1}$ vibrational energy levels are more intense and are drawn using solid lines. The less intense $A_{2}$ levels are drawn using dashed lines. The symmetry designations denote the symmetry of the wavefunctions across the center of the wells. The intermediate to high-lying vibrational energy levels computed with the geometric phase (GP) are significantly different than those computed without the geometric phase (NGP) (see text for discussion). 
B. Kendrick, Fig. 1

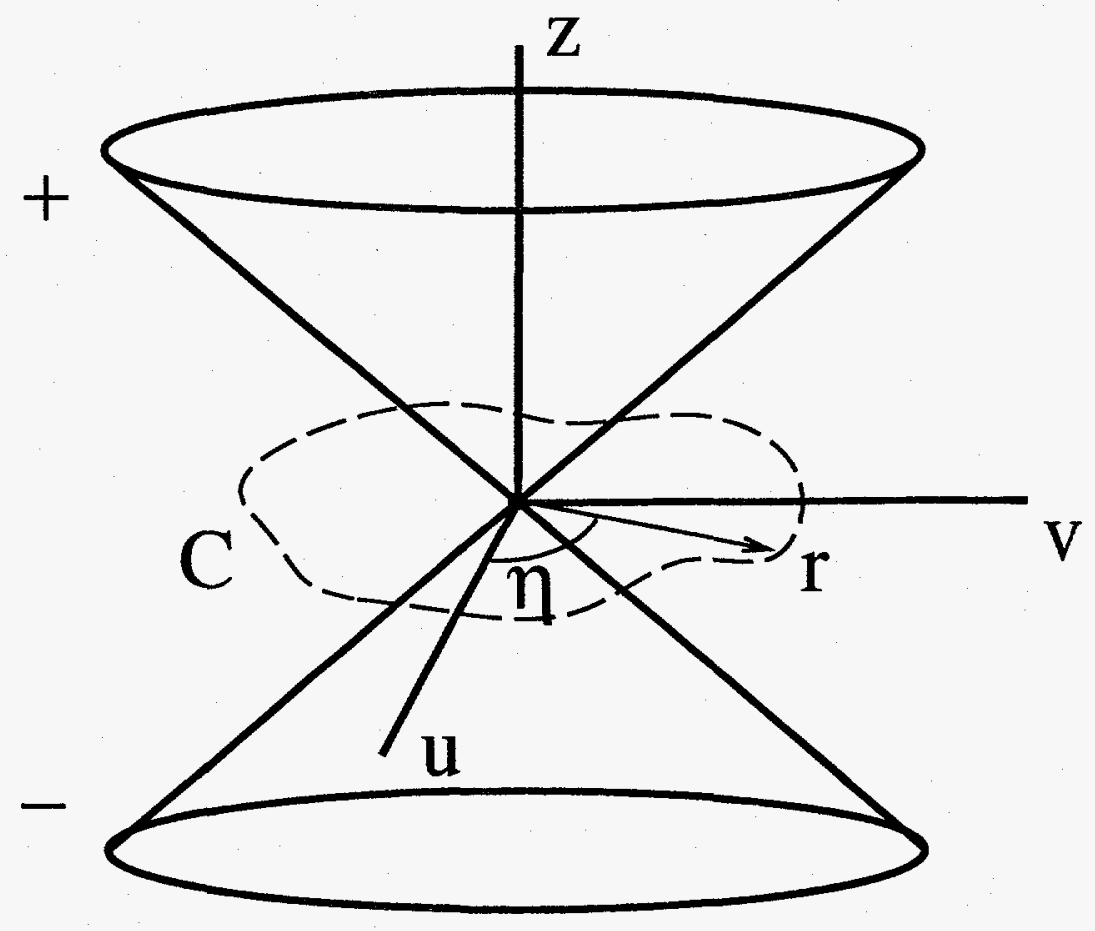


B. Kendrick, Fig. 2

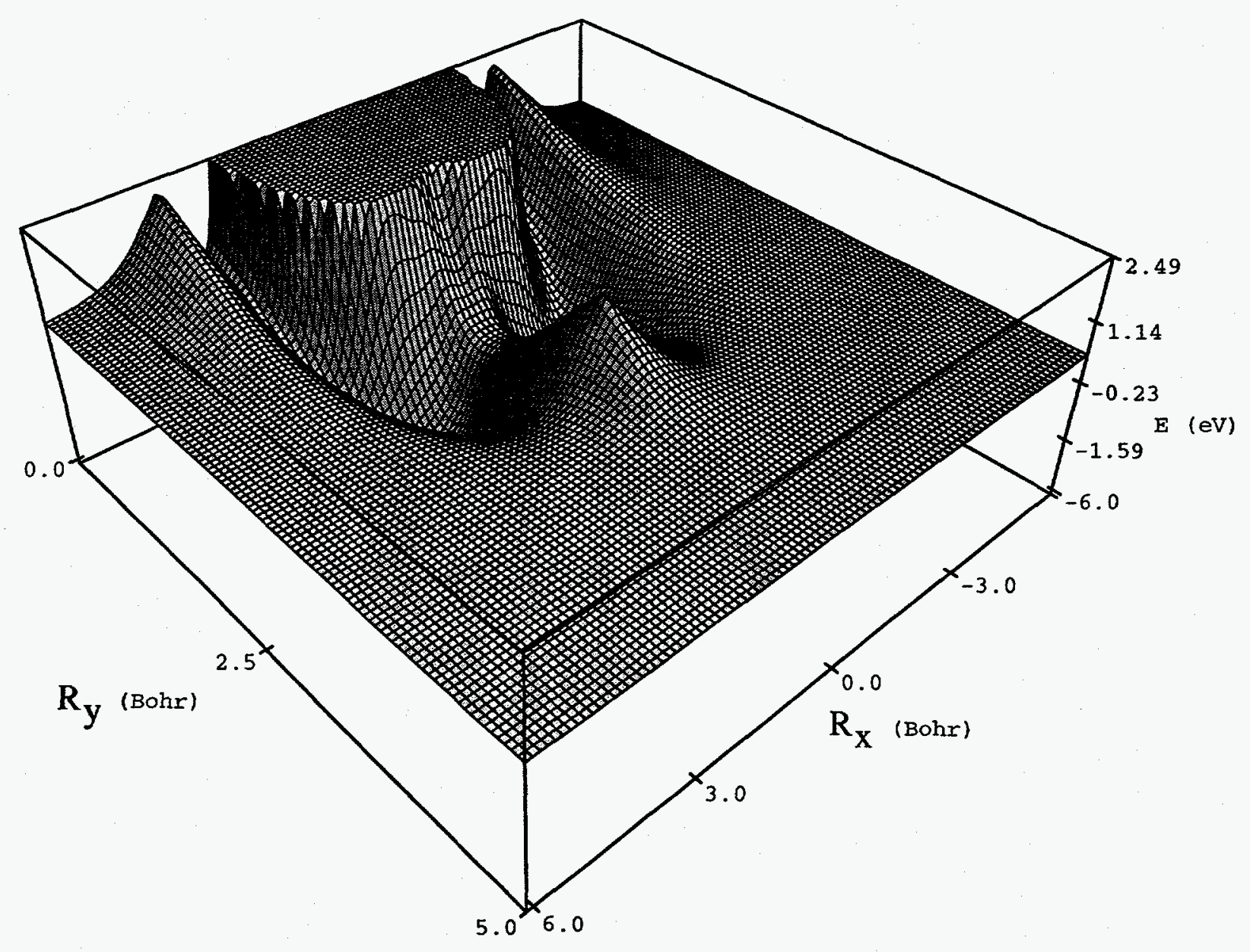


B. Kendrick, Fig. 3

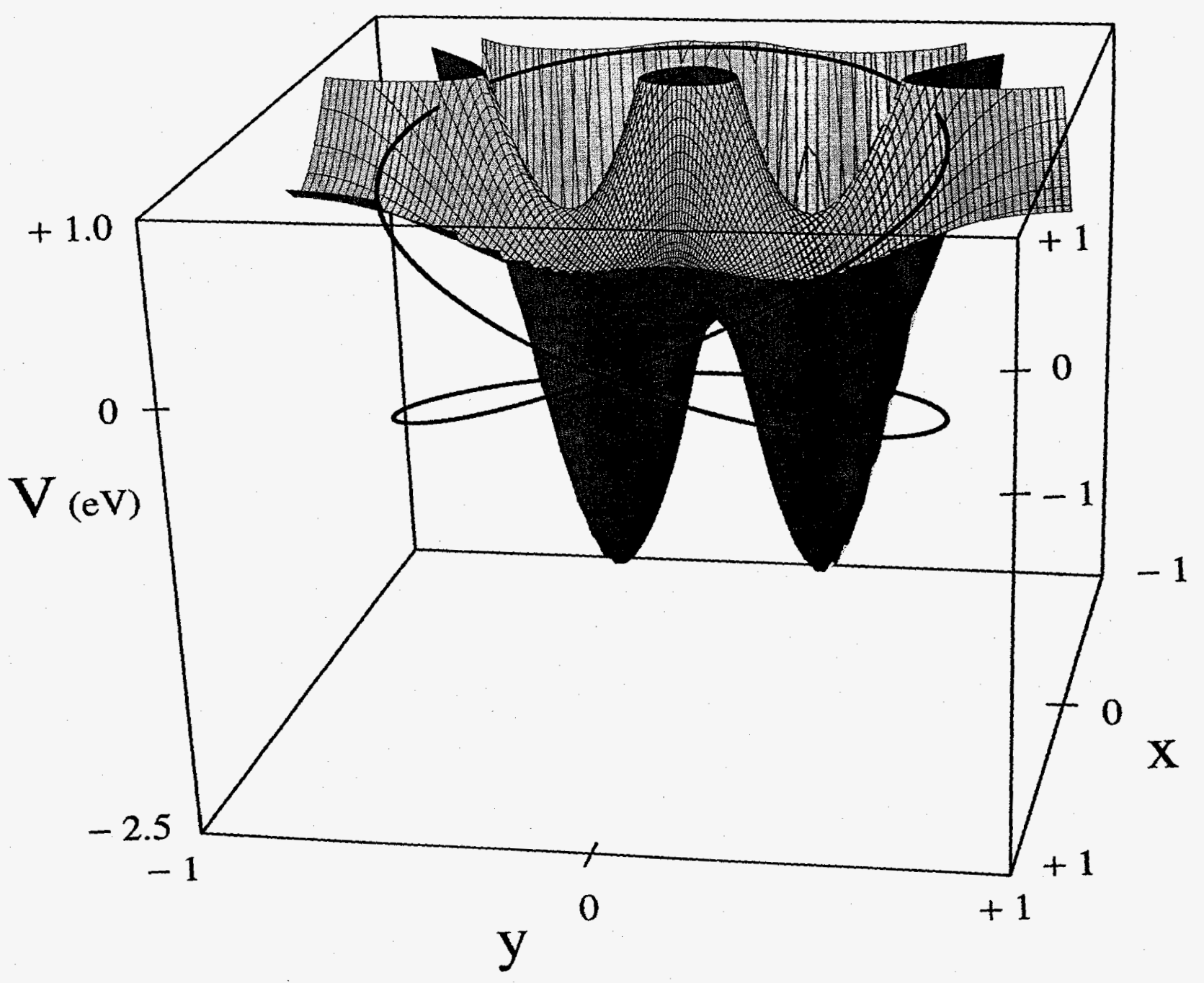


B. Kendrick, Fig. 4
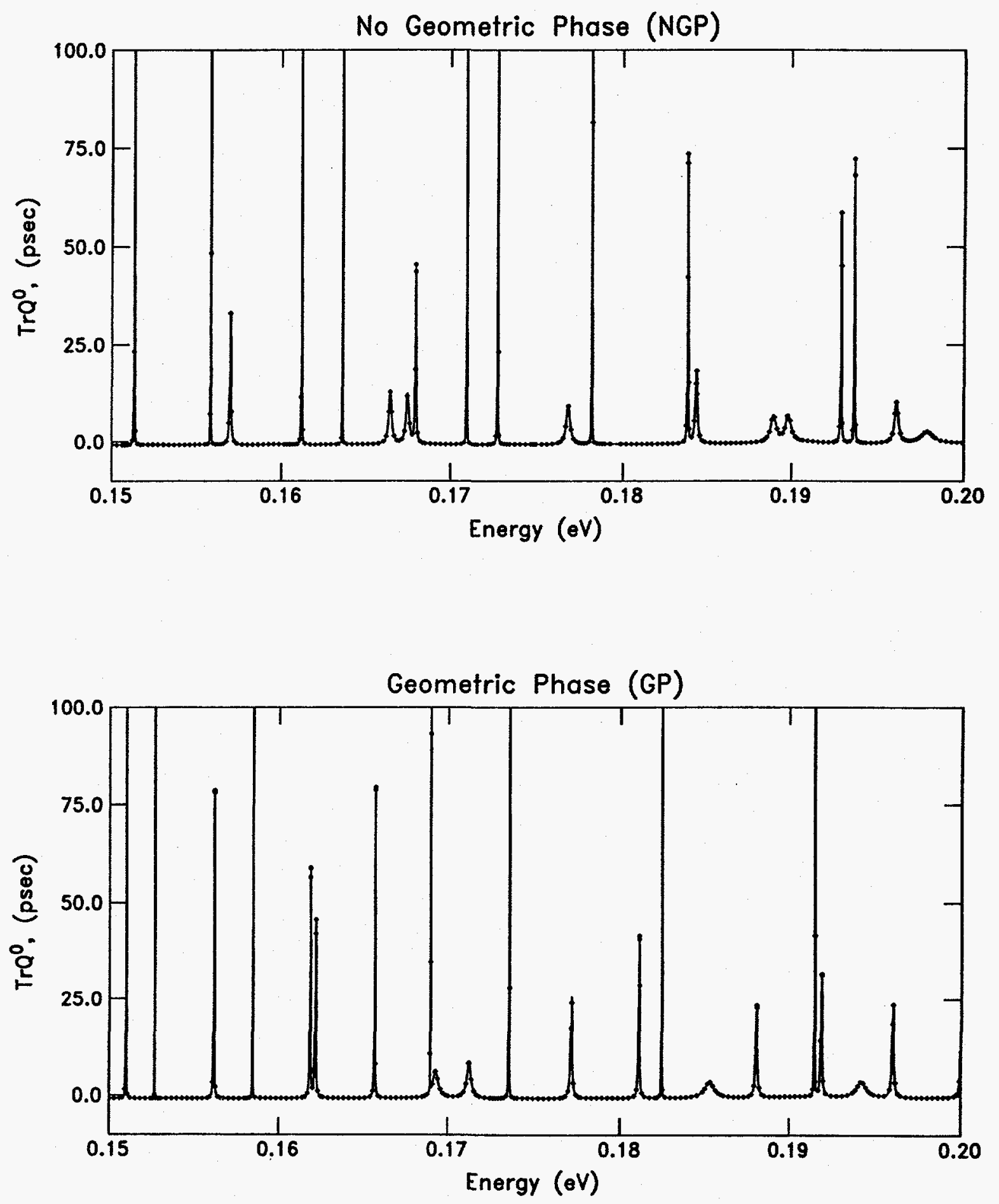
B. Kendrick, Fig. 5
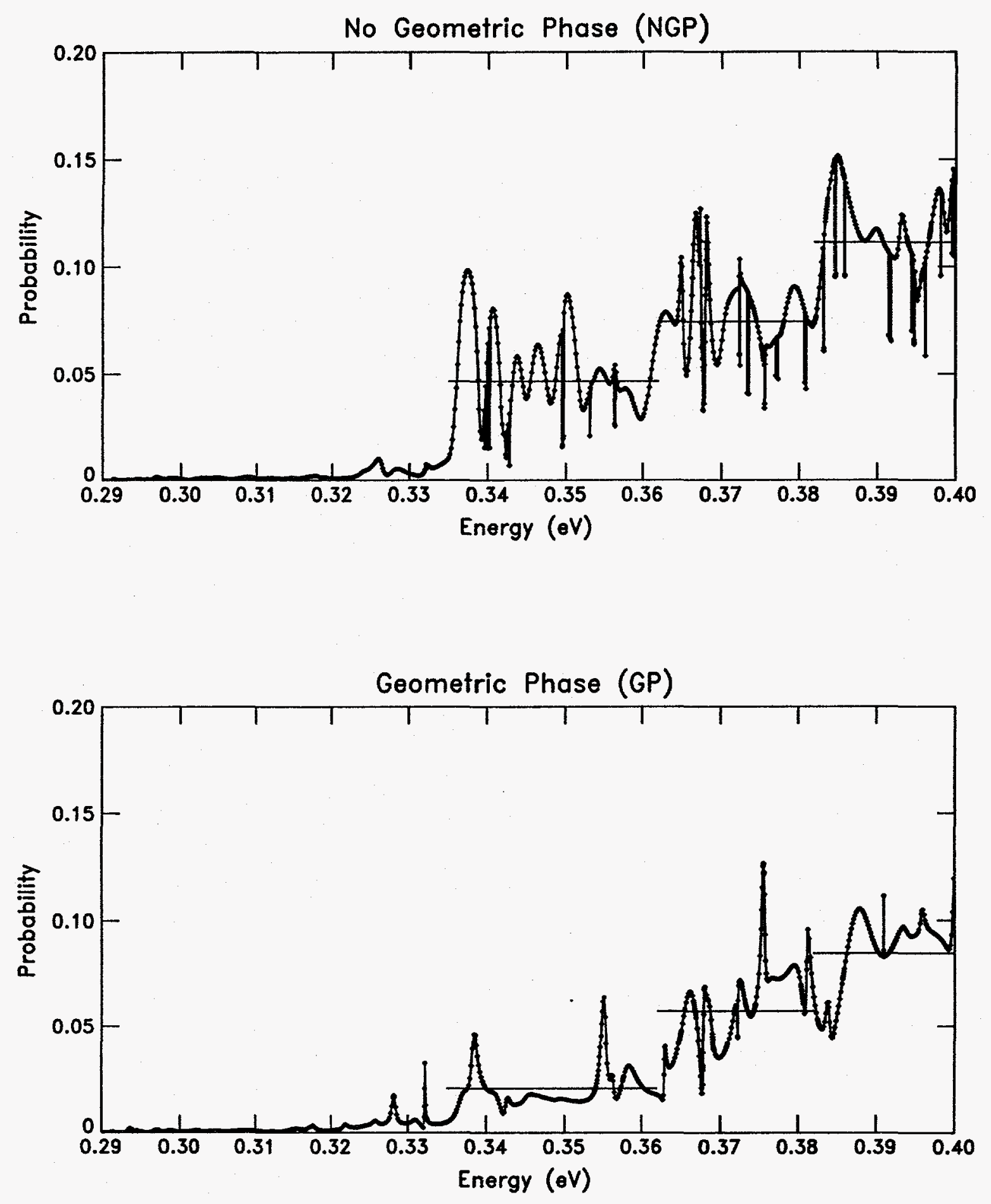
B. Kendrick, Fig. 6

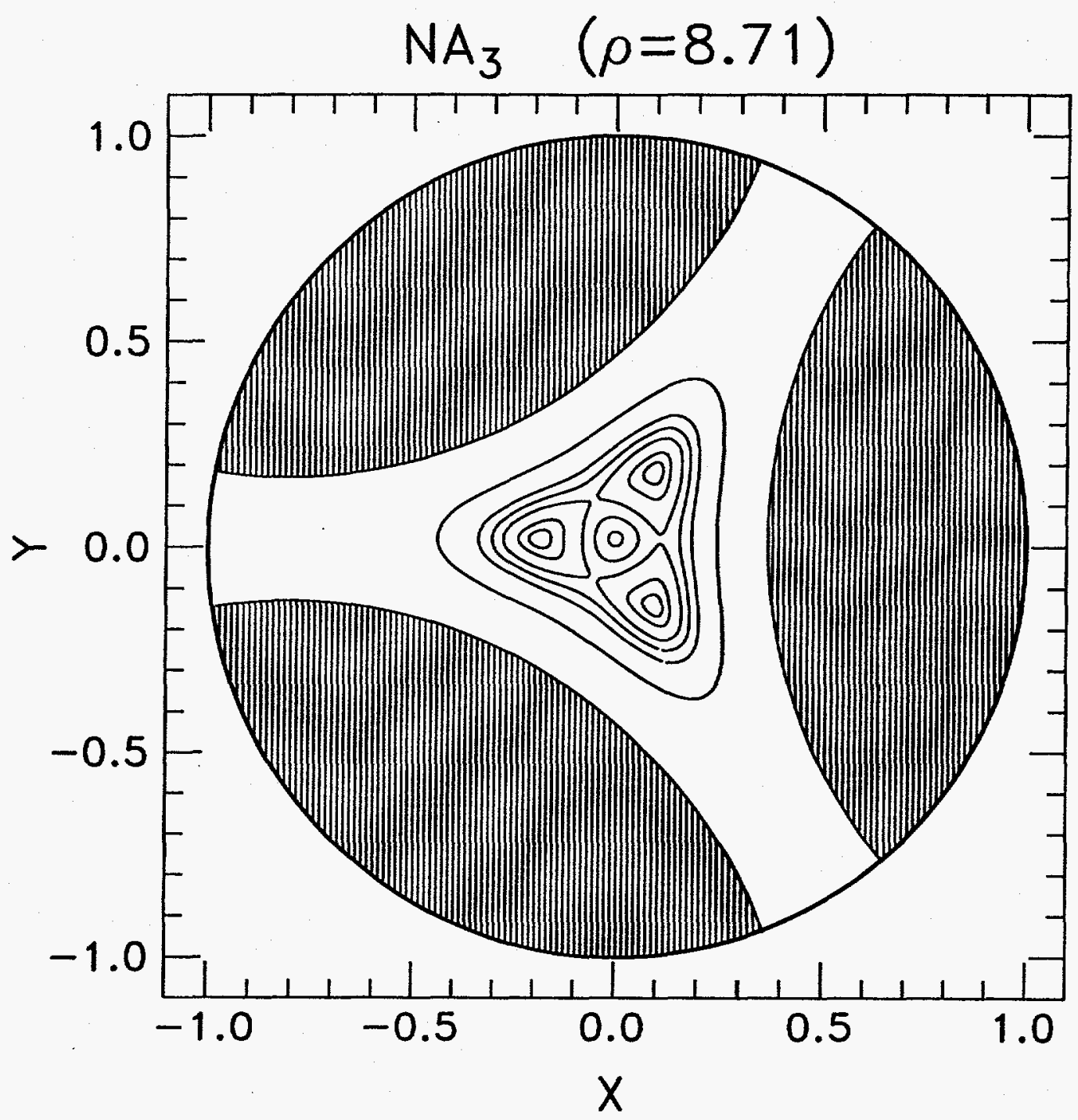


B. Kendrick, Fig. 7

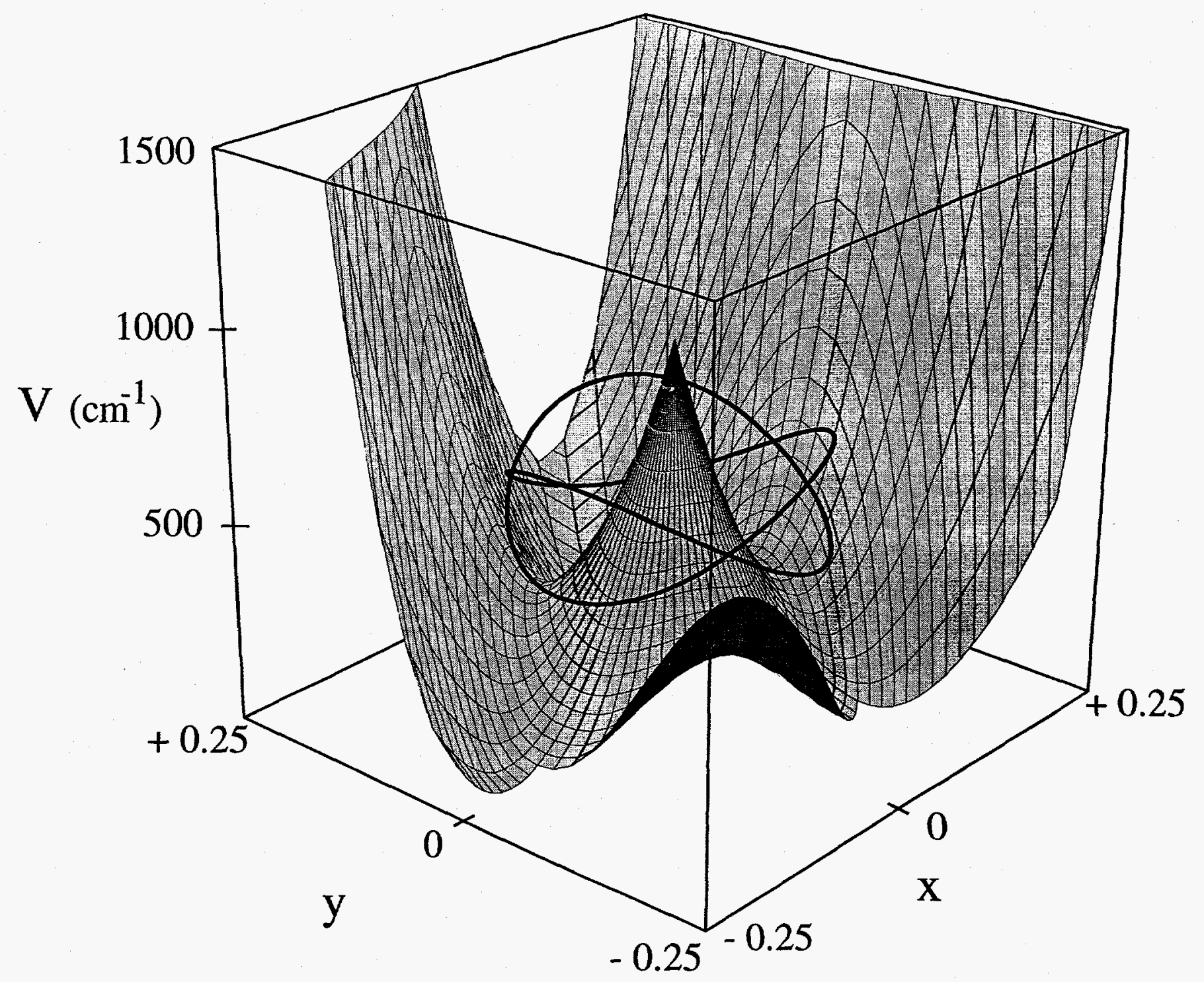


B. Kendrick, Fig. 8

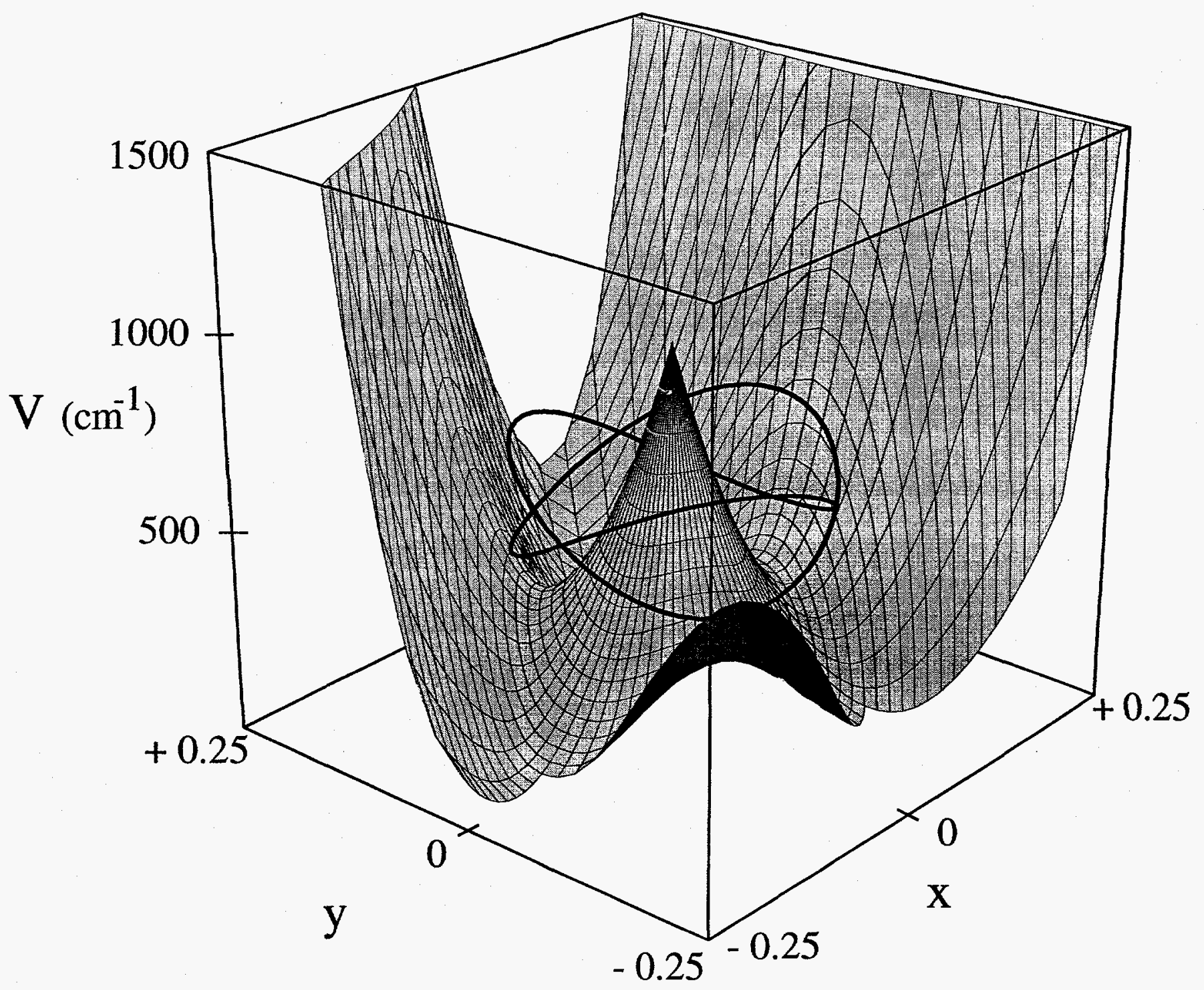


B. Kendrick, Fig. 9

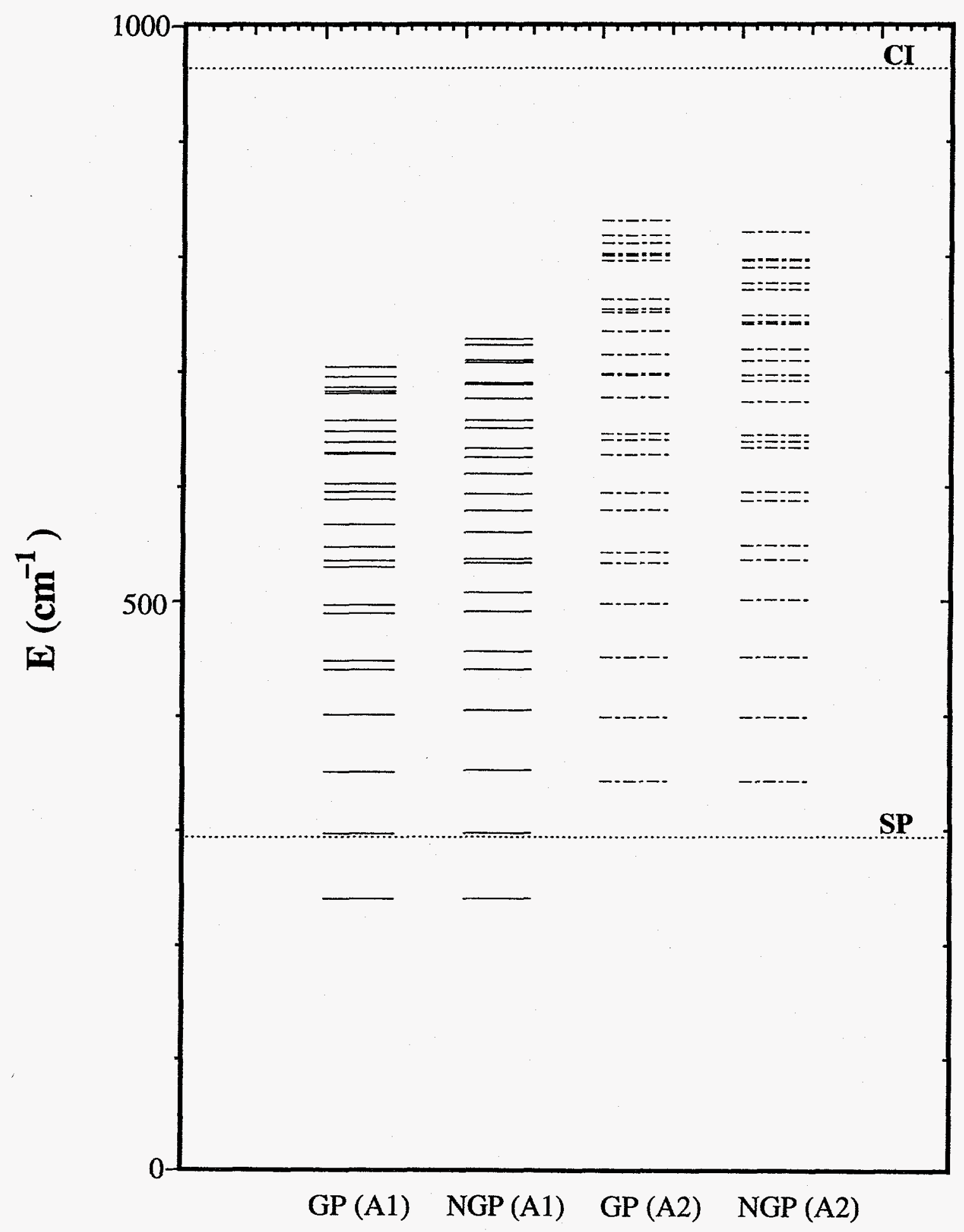

